\title{
New Conjunctive Reagents as Cross-Coupling Partners en route to Retinoid-like Polyenes
}

Bruce H. Lipshutz, ${ }^{\text {aa }}$ Giuliano C. Clososki, ${ }^{a}$ Will Chrisman, ${ }^{a}$ David W. Chung, ${ }^{a}$ David B. Ball, ${ }^{b}$ and Jennifer Howell ${ }^{b}$

\author{
${ }^{a}$ Department of Chemistry and Biochemistry, \\ University of California, Santa Barbara, CA 93106 and \\ ${ }^{b}$ Department of Chemistry, California State University \\ Chico, CA 95929
}

Supporting Information 
General. All experiments were conducted under an argon atmosphere, in oven dried glassware with magnetic stirring, unless otherwise specified. Handling of polyenes was done under photographic darkroom lights type 'YG10-gold' for light cutoff at $475 \mathrm{~nm}$, purchased from Encapsulight of Hayward, CA.; TEL: 1-888-274-8626. Reagents obtained from commercial sources were purified before use unless otherwise noted, except organometallic reagents which were used as received from Aldrich in Sure-Seal bottles. Chromatography (TLC) was performed using EM Science 60-F 254 (250 nm) silica gel precoated plates; EM Science cat. No. 5714-3. Flash chromatography was performed using silica gel; 200-425 mesh, 60A Grade 633 available from Fisher Scientific. Flash chromatography columns were slurry packed. FT-IR spectra were recorded using a Mattson Infinity M60t instrument with either neat samples on $\mathrm{NaCl}$ plates, $\mathrm{KBr}$ pellets, or dilute chloroform solutions. HRMS spectra were recorded at the UCSB mass spectrometry facility by Dr. James Pavlovich using a Micromass VG 70e magnetic sector by EI, CI (using $\mathrm{CH}_{4}$ ), or FAB (using NBA) standard methods. NMR spectra were recorded using Varian Inova spectrometers either at 400 or $500 \mathrm{MHz}$ for ${ }^{1} \mathrm{H}$, with dilute solutions in $\mathrm{CDCl}_{3}$ or DMSO-d 6 (3 mm or $5 \mathrm{~mm}$ tubes $)$. Chemical shifts are referenced to either TMS or recognized solvent peaks. Melting points were measured in duplicate, using a Melt-Temp apparatus; the reported values are referenced to benzoic acid, CAS No. [65-85-0]; lit. $\mathrm{mp}=121-123{ }^{\circ} \mathrm{C}$, observed $\mathrm{mp}=$ $120-122.5^{\circ} \mathrm{C}$.

(E)-3-(Tributylstannyl)but-2-en-1-ol (E-11). A flame dried $500 \mathrm{~mL}$ round bottom flask fitted with a heavy Teflon-coated stir bar was cooled under a stream of argon and charged with $\mathrm{CuCN}$ (4.51 g, 50.4 mmol). THF (150 mL) was added and the flask was fitted with a rubber septum. While stirring under argon the solution was cooled to $-78{ }^{\circ} \mathrm{C}$ and treated with $n$-BuLi $(2.65 \mathrm{M}$, hexanes, $40.1 \mathrm{~mL}, 0.106$ mol). The solution was warmed to $-20{ }^{\circ} \mathrm{C}$ for 3 min then recooled to $-78{ }^{\circ} \mathrm{C}$. This warming and cooling cycle was repeated until a homogeneous cuprate $\mathrm{Bu}_{2} \mathrm{Cu}(\mathrm{CN}) \mathrm{Li}_{2}$ was formed. The $-78{ }^{\circ} \mathrm{C}$ cuprate solution was treated with tri- $n$-butylstannyl hydride $(29.2 \mathrm{~mL}, 0.109 \mathrm{~mol}$, neat $)$ which was accompanied by gas evolution. Over a period of $45 \mathrm{~min}$ an orange colored solution formed. To the $-78{ }^{\circ} \mathrm{C}$ tri- $n$ - 
butylstannylcuprate was added 2-butyn-1-ol (3.71 mL, $49.5 \mathrm{mmol}$, neat) over $10 \mathrm{~min}$ with stirring. The reaction was continued for $2 \mathrm{~h}$ at $-78{ }^{\circ} \mathrm{C}$ and quenched at cold temp., by fast addition of dry methanol (5.0 mL, $123.2 \mathrm{mmol})$. Saturated aq. $\mathrm{NH}_{4} \mathrm{Cl}$ was added $(100 \mathrm{~mL})$ and the stirred solution was warmed to $\mathrm{rt}$ over $2-3 \mathrm{~h}$. The mixture was then poured into a separatory funnel containing water $(50 \mathrm{~mL})$ and extracted using $\mathrm{Et}_{2} \mathrm{O}(3 \times 50 \mathrm{~mL})$. The combined organic layer was washed using water, and brine, and dried over anhydrous $\mathrm{MgSO}_{4}$. The dried organics were concentrated under reduced pressure and a yellow oil was obtained. The crude product was loaded on silica gel and eluted with $\mathrm{Et}_{2} \mathrm{O}$-hexanes 1:4. Purified $\boldsymbol{E}$-11, $\mathrm{R}_{\mathrm{f}}=0.35$ (Seebach stain), $\mathrm{Et}_{2} \mathrm{O}$-hexanes 1:4, was obtained $(14.52 \mathrm{~g}, 80 \%)$ as a yellow oil. ${ }^{1} \mathrm{H}$ NMR (400 MHz, $\left.\mathrm{CDCl}_{3}\right) \delta 5.76\left(\mathrm{~m},{ }^{3} J_{\mathrm{SnH}}=33.7 \mathrm{~Hz}\right.$, vinyl-H), 4.26 (app. dd, $J=0.82,6.2 \mathrm{~Hz}$, 2H), 1.89 (app. d, $J=1.2 \mathrm{~Hz},{ }^{3} J_{\mathrm{SnH}}=22.7 \mathrm{~Hz}, \mathrm{CH}_{3}$ ), $1.53-1.43(\mathrm{~m}, 7 \mathrm{H}), 1.33$ (sextet, $J=7.2 \mathrm{~Hz}, 6 \mathrm{H}$ ), 0.91-0.87 (m, 15H); ${ }^{13} \mathrm{C}$ NMR $\left(100 \mathrm{MHz}, \mathrm{CDCl}_{3}\right) \delta 142.7,139.4,59.1,29.2,27.6,19.6,13.9,9.3 ;$ IR (NaCl, neat) 3307, 2925, 1463, 1059, 1004, 864, 663, $595 \mathrm{~cm}^{-1}$; MS (m/z, rel \%) 57(32), 75(21), 97(4), 121(51), 137(55), 149(6), 177(42), 193(68), 203(5), 235(6), 235(6), 249(64), 291(2), 305 (100), 319(4); HREI m/z calcd. for $\mathrm{C}_{12} \mathrm{H}_{25} \mathrm{OSn}: 301.0923\left({ }^{116} \mathrm{Sn}, \mathrm{M}-\mathrm{C}_{4} \mathrm{H}_{9}\right)^{+}$, found: 301.0922 .

(E)-3-(Tributylstannyl)but-2-en-1-al (12). A $250 \mathrm{~mL}$ round bottom flask fitted with a heavy Tefloncoated stir bar was charged with $(E)-3$-tributylstannyl-but-2-en-1-ol $(\boldsymbol{E}-11,10.18 \mathrm{~g}, 28.1 \mathrm{mmol})$ and $\mathrm{CH}_{2} \mathrm{Cl}_{2}$ was added $(125 \mathrm{~mL})$. With stirring, small portions of manganese(IV) oxide ( $88 \%$ active $\mathrm{MnO}_{2}$ ) totaling $48.0 \mathrm{~g}$ were added in ca. $6 \mathrm{~h}$ intervals. The disappearance of $\boldsymbol{E}-\mathbf{1 1}, \mathrm{R}_{\mathrm{f}}=0.16$ (iodine stain), $\mathrm{Et}_{2} \mathrm{O}$-hexanes 1:8, was monitored by TLC and the reaction was stopped at $24 \mathrm{~h}$. The stannylaldehyde product, but not the starting material, was visualized with a UV lamp, $\mathrm{R}_{\mathrm{f}}=0.50$ (iodine stain), $\mathrm{Et}_{2} \mathrm{O}$ hexanes 1:8. The solution was filtered on paper using liberal quantities of $\mathrm{CH}_{2} \mathrm{Cl}_{2}$ to wash the filter cake. The combined organic layers were concentrated under reduced pressure leaving a yellow oil. The crude product was loaded on silica gel and eluted with $\mathrm{Et}_{2} \mathrm{O}$-hexanes 1:8. Purified 12, $\mathrm{R}_{\mathrm{f}}=0.45$ (iodine stain), $\mathrm{Et}_{2} \mathrm{O}$-hexane $1: 8$, was obtained $(8.37 \mathrm{~g}, 83 \%)$ as a yellow oil. ${ }^{1} \mathrm{H}$ NMR $\left(400 \mathrm{MHz}, \mathrm{CDCl}_{3}\right) \delta$ $10.05\left(\mathrm{~d}, J=7.9 \mathrm{~Hz},{ }^{3} J_{\mathrm{SnH}}=87.5 \mathrm{~Hz}, \mathrm{CHO}\right), 6.11$ (app. dd, $J=1.9,7.9 \mathrm{~Hz},{ }^{3} J_{\mathrm{SnH}}=87.5 \mathrm{~Hz}$, vinyl-H), 
2.42 (app. d, $\left.J=1.2 \mathrm{~Hz},{ }^{3} J_{\mathrm{SnH}}=22.7 \mathrm{~Hz}, \mathrm{CH}_{3}\right), 1.49(\mathrm{~m}, 6 \mathrm{H}), 1.30(\mathrm{~m}, 6 \mathrm{H}), 0.98(\mathrm{~m}, 6 \mathrm{H}), 0.41(\mathrm{~m}$, $9 \mathrm{H}) ;{ }^{13} \mathrm{C} \mathrm{NMR}\left(100 \mathrm{MHz}, \mathrm{CDCl}_{3}\right) \delta 187.6,174.5,140.1,29.13,27.5,20.9,13.8,9.6$; IR $(\mathrm{NaCl}$, neat): 2927, 2853, 1674, 1462, 1144, 1062, 934, 674; MS (m/z, rel \%): 53(7), 69(4), 121(35), 137(13), 159 (15), 177(5), 159(15), 177(26), 189(8), 247(43), 303(100); HREI m/z calcd. for $\mathrm{C}_{12} \mathrm{H}_{23} \mathrm{OSn}: 299.0766$ $\left({ }^{116} \mathrm{Sn}, \mathrm{M}-\mathrm{C}_{4} \mathrm{H}_{9}\right)^{+}$, found: 299.0773 .

Tri-n-Butyl-(3-trimethylsilyl-2-propynyl)phosphonium bromide (13). A flame dried $300 \mathrm{~mL}$ round bottom flask fitted with a heavy Teflon-coated stir bar was cooled under a stream of argon and charged with Fluka Chemicals cat. No. 92724, pract. grade (95\%), 3-(trimethylsilyl)propargyl bromide (22.8 g, ca. $119.3 \mathrm{mmol})$ and benzene $(115 \mathrm{~mL})$, then topped with a rubber septum. The solution was cooled to $0{ }^{\circ} \mathrm{C}$ under argon and treated dropwise with freshly distilled tri- $n$-butylphosphine $(47.6 \mathrm{~mL}, 191.0$ mmol, neat) over $20 \mathrm{~min}$. The solution gradually became yellow, then dark orange. After $30 \mathrm{~min}$, the ice-water bath was removed and the mixture was stirred at rt overnight. The solvent and excess tri- $n$ butylphosphine were stripped under reduced pressure leaving an amber residue. The residue was triturated by distilled hexanes, and the precipitate was filtered on a Buchner funnel under argon. The filter cake was washed with hexanes $(3 \times 40 \mathrm{~mL})$, and then dried at $\mathrm{rt}$ in a vacuum desiccator $(1.0 \mathrm{~mm}$ $\mathrm{Hg}$ ) overnight. Purified 13 was obtained (46 g, 98\%) as a white solid; mp $=118.5-120{ }^{\circ} \mathrm{C} .{ }^{1} \mathrm{H}$ NMR $\left(500 \mathrm{MHz}, \mathrm{CDCl}_{3}\right) \delta 3.91$ (app. d, $J=15.3 \mathrm{~Hz}, \mathrm{CH}_{2}$ ), 2.6-2.54 (m, 6H), 1.62-1.58 (m, 6H), 1.52-1.49 $(\mathrm{m}, 6 \mathrm{H}), 0.96$ (app. t, $J=7.0 \mathrm{~Hz}, 9 \mathrm{H}) ; 0.14(\mathrm{~s}, 9 \mathrm{H}) ;{ }^{13} \mathrm{C} \mathrm{NMR}\left(125 \mathrm{MHz}, \mathrm{CDCl}_{3}\right) \delta 92.97\left(\mathrm{~d},{ }^{3} J_{\mathrm{PC}}=\right.$ $12.8 \mathrm{~Hz}), 92.65\left(\mathrm{~d},{ }^{2} J_{\mathrm{PC}}=5.5 \mathrm{~Hz}\right), 24.1\left(\mathrm{~d},{ }^{3} J_{\mathrm{PC}}=16.5 \mathrm{~Hz}\right), 23.92\left(\mathrm{~d},{ }^{4} J_{\mathrm{PC}}=3.6 \mathrm{~Hz}\right), 19.22\left(\mathrm{~d},{ }^{1} J_{\mathrm{PC}}=\right.$ $45.8 \mathrm{~Hz}), 14.25\left(\mathrm{~d},{ }^{1} J_{\mathrm{PC}}=51.2 \mathrm{~Hz}, \mathrm{CH}_{2}\right), 13.62(\mathrm{~s}),-0.28(\mathrm{~s}, \mathrm{TMS})$. HRMS (FAB-NBA) m/z calcd. for $\mathrm{C}_{18} \mathrm{H}_{38} \mathrm{SiP} 313.2480(\mathrm{M}-\mathrm{Br})^{+}$, found 313.2472.

[(3E,5E)-6-(Tributylstannyl)hepta-3,5-dien-1-ynyl]trimethylsilane $(E-2)$. A flame dried $1 \mathrm{~L}$ round bottom flask fitted with a heavy Teflon-coated stir bar was cooled under a stream of argon and charged with tri- $n$-butyl-(3-trimethylsilyl-2-propynyl)phosphonium bromide (13, $9.31 \mathrm{~g}, 23.7 \mathrm{mmol})$ and THF $(600 \mathrm{~mL})$. The flask was topped with a rubber septum and cooled under argon to between -5 to $0{ }^{\circ} \mathrm{C}$, 
then treated with sodium bis(trimethylsilyl)amide (1.0 M, THF) (25.0 mL) with stirring giving an amber heterogeneous solution. The stirred mixture was warmed to $\mathrm{rt}$ over $40 \mathrm{~min}$, then cooled to between -105 to $-99{ }^{\circ} \mathrm{C}$ in a pentane-liquid nitrogen bath. A precooled solution of $(E)-3-$ tributylstannyl-but-2-en-1-al (12, $7.92 \mathrm{~g}, 22.1 \mathrm{mmol})$ in THF (150 mL) was transferred dropwise at a rate of about $1.0 \mathrm{~mL} / \mathrm{min}$ to the phosphonium salt using a precooled cannula, with vigorous stirring and careful temperature regulation (as needed adding portions of liquid nitrogen to the cooling bath). After $2 \mathrm{~h}$ at this cold temp., the work-up was begun by rapid addition of ice-cold propylene oxide $(5.0 \mathrm{~mL})$ followed by trimethylchlorosilane $(5.0 \mathrm{~mL})$. The cooling bath was removed and the solution was stirred while warming over $25 \mathrm{~min}$, then poured into a $1 \mathrm{~L}$ separatory funnel containing sat. aq. $\mathrm{NaHCO}_{3}(150$ $\mathrm{mL}$ ) and extracted using hexanes (3 x $140 \mathrm{~mL})$. The combined organic layers were washed using water, and brine, and then dried over anhydrous $\mathrm{Na}_{2} \mathrm{SO}_{4}$. The dried organics were concentrated under reduced pressure leaving to a dark oil. The crude product was loaded on silica gel (doped with triethylamine 3\% v/v) and eluted with hexanes. Purified $(E, E)$-stannyldienyne $\boldsymbol{E}-2, \mathrm{R}_{\mathrm{f}}=0.52$ (PMA stain), hexanes, was obtained (7.21 g, 71\%) as a yellow oil; ${ }^{1} \mathrm{H}$ NMR $\left(500 \mathrm{MHz}, \mathrm{CDCl}_{3}\right) \delta 6.92($ app dd, $J=11.0,15.6 \mathrm{~Hz}$, 1H), 6.23 (app. dd, $\left.J=2.1,11.3 \mathrm{~Hz},{ }^{3} J_{\mathrm{SnH}}=32.3 \mathrm{~Hz}, 1 \mathrm{H}\right), 5.53$ (d, $\left.J=15.6 \mathrm{~Hz}, 1 \mathrm{H}\right), 2.01$ (app. d, $J=$ $\left.1.8 \mathrm{~Hz},{ }^{3} J_{\mathrm{SnH}}=23.4 \mathrm{~Hz}, \mathrm{CH}_{3}\right), 1.49(\mathrm{~m}, 6 \mathrm{H}), 1.29(\mathrm{~m}, 6 \mathrm{H}), 1.22(\mathrm{~m}, 6 \mathrm{H}), 0.90(\mathrm{~m}, 9 \mathrm{H}), 0.20(\mathrm{~s}, 9 \mathrm{H})$; ${ }^{13} \mathrm{C} \mathrm{NMR}\left(125 \mathrm{MHz}, \mathrm{CDCl}_{3}\right) \delta 150.4$ (quat.), $138.2\left({ }^{3} J_{\mathrm{SnC}}=10.1 \mathrm{~Hz}\right), 137.2\left({ }^{2} J_{\mathrm{SnC}}=27.2 \mathrm{~Hz}\right), 109.7$, 105.4 (alkyne), 97.4 (alkyne), 29.3, 27.5, $20.5\left(\mathrm{CH}_{3}\right)$, 13.9, 9.2, 0.2 (TMS); IR (NaCl, neat): 2957 , 2926, 2218, 1462, 1248, $843 \mathrm{~cm}^{-1}$; MS (m/z, rel \%) 73(42), 121(20), 135(34), 177(16), 285(32), 341 (30), 397(100), 439(30), 454(2). HREI m/z calcd. for $\mathrm{C}_{18} \mathrm{H}_{33} \mathrm{SiSn}: 393.1369\left({ }^{116} \mathrm{Sn}, \mathrm{M}-\mathrm{C}_{4} \mathrm{H}_{9}\right)^{+}$, found: 393.1370.

(Z)-3-Tributylstannyl-but-2-en-1-ol (Z-11). A flame dried $500 \mathrm{~mL}$ round bottom flask fitted with a heavy Teflon-coated stir bar was cooled under a stream of argon, charged with 2-butyn-1-ol (10; $2.80 \mathrm{~g}$, $39.9 \mathrm{mmol})$ and THF $(175 \mathrm{~mL})$. The flask was topped with a rubber septum, stirred at $\mathrm{rt}$ under an argon, and treated dropwise with Red-Al (16.0 mL, $44 \mathrm{mmol})$; the initial volume of Red-Al(ca. 8.0 
$\mathrm{mL}$ ) was added dropwise due to gas evolution, while the balance was added fairly quickly once gas evolution had stopped. The solution was stirred for $1.5 \mathrm{~h}$ then treated with tri- $n$-butylstannyl chloride (23.1 $\mathrm{mL}, 85.2 \mathrm{mmol}$, neat) over $1 \mathrm{~min}$. The reaction was continued for $18-20 \mathrm{~h}$, then quenched by addition of sat. sodium tartrate (aq) $(120 \mathrm{~mL})$. The mixture was stirred for $3 \mathrm{~h}$ then poured into a separatory funnel containing water $(50 \mathrm{~mL})$ plus sat. aq. sodium tartrate $(50 \mathrm{~mL})$, and extracted using $\mathrm{Et}_{2} \mathrm{O}(3 \times 60 \mathrm{~mL})$. The combined organic layers were washed using water, and brine, and then dried over anhydrous $\mathrm{MgSO}_{4}$. The dried organics were concentrated under reduced pressure and a yellow oil was obtained. The crude produuct was loaded on silica gel and eluted with $\mathrm{Et}_{2} \mathrm{O}$ :hexanes 1:5. Purified hydroxyvinylstannane $\boldsymbol{Z}-11, \mathrm{R}_{\mathrm{f}}=0.36$ (PMA stain), $\mathrm{Et}_{2} \mathrm{O}$ :hexanes 1:5, was obtained $(9.4 \mathrm{~g}, 65 \%$ ) as a yellow oil. ${ }^{1} \mathrm{H}$ NMR (400 MHz, $\left.\mathrm{CDCl}_{3}\right) \delta 6.27$ (app td, $J=1.6,6.7, \mathrm{~Hz},{ }^{3} J_{\mathrm{SnH}}=62.1 \mathrm{~Hz}$, vinyl-H), 4.02 (app. t, $J=5.6 \mathrm{~Hz}, 2 \mathrm{H}), 1.95$ (app. d, $\left.J=1.7 \mathrm{~Hz},{ }^{3} J_{\mathrm{SnH}}=23.5 \mathrm{~Hz}, \mathrm{CH}_{3}\right), 1.48(\mathrm{~m}, 6 \mathrm{H}), 1.31(\mathrm{~m}, 6 \mathrm{H})$, $0.93(\mathrm{~m}, 6 \mathrm{H}), 0.89(\mathrm{~m}, 9 \mathrm{H}) ;{ }^{13} \mathrm{C} \mathrm{NMR}\left(100 \mathrm{MHz}, \mathrm{CDCl}_{3}\right) \delta$ 144.6, 139.0, 65.2, 29.3, 27.6, 27.2, 13.9, 10.4; IR (NaCl, neat) 3337, 2957, 2926, 2871, 2853, 1461, 1068, 1000, $665 \mathrm{~cm}^{-1}$; MS (m/z, rel \%): 71(43), 121(24), 137(45), 177(51), 191(16), 269(6), 305(100). HREI m/z calcd. for $\mathrm{C}_{12} \mathrm{H}_{25} \mathrm{OSn}$ $301.0923\left({ }^{116} \mathrm{Sn}, \mathrm{M}-\mathrm{C}_{4} \mathrm{H}_{9}\right)^{+}$, found 301.0915 .

(Z)-3-Tributylstannyl-but-2-en-1-al. A $250 \mathrm{~mL}$ round bottom flask fitted with a heavy Teflon-coated stir bar was charged with (Z)-3-tributylstannyl-but-2-en-1-ol (Z-11, $9.7 \mathrm{~g}, 26.9 \mathrm{mmol})$ and $\mathrm{CH}_{2} \mathrm{Cl}_{2}(125$ $\mathrm{mL}$ ). With stirring, small portions of manganese(IV) oxide ( $88 \%$ active $\mathrm{MnO}_{2}$ ) totaling $43.0 \mathrm{~g}$ were added in ca. $6 \mathrm{~h}$ intervals. The disappearance of $\boldsymbol{Z - 1 1}, \mathrm{R}_{\mathrm{f}}=0.45$ (iodine stain), $\mathrm{Et}_{2} \mathrm{O}$-hexanes 1:5, was monitored by TLC and the reaction was stopped after $48 \mathrm{~h}$. The stannylaldehyde product, but not the starting material, was visualized with a UV lamp, $\mathrm{R}_{\mathrm{f}}=0.83, \mathrm{Et}_{2} \mathrm{O}$-hexanes 1:5. The solution was filtered on paper using liberal quantities of $\mathrm{CH}_{2} \mathrm{Cl}_{2}$ to wash the filter cake. The combined organic layers were concentrated under reduced pressure and a yellow oil was obtained. The crude product was loaded on silica gel and eluted with EtOAc:hexanes 1:25. Purified Z-enal, $\mathrm{R}_{\mathrm{f}}=0.42$ (iodine stain), EtOAc:hexanes 1:25, was obtained ( $7.43 \mathrm{~g}, 77 \%$ ) as a yellow oil. ${ }^{1} \mathrm{H}$ NMR (400 MHz, $\left.\mathrm{CDCl}_{3}\right) \delta 9.46$ (app. d, $J=6.9$ 
$\mathrm{Hz}, \mathrm{CHO}$ ), 6.68 (app. dd, $J=1.5,6.9 \mathrm{~Hz},{ }^{3} J_{\mathrm{SnH}}=50.6 \mathrm{~Hz}$, vinyl-H), 2.23 (app. d, $J=1.6 \mathrm{~Hz},{ }^{3} J_{\mathrm{SnH}}=$ $\left.18.9 \mathrm{~Hz}, \mathrm{CH}_{3}\right), 1.48(\mathrm{~m}, 6 \mathrm{H}), 1.29(\mathrm{~m}, 6 \mathrm{H}), 1.10(\mathrm{~m}, 6 \mathrm{H}), 0.84(\mathrm{~m}, 9 \mathrm{H}) ;{ }^{13} \mathrm{C} \mathrm{NMR}\left(100 \mathrm{MHz}, \mathrm{CDCl}_{3}\right) \delta$ 193.7, 179.3, 141.6, 29.2, 28.8, 27.4, 13.8, 11.2; IR (NaCl, neat) 2956, 2926, 2851, 1687, 1564, 1461, 1152, 758, 669, $594 \mathrm{~cm}^{-1}$; MS (m/z, rel \%) 53(7), 121(14), 137(11), 161(13), 177(9), 189(24), 247(7), 303(100); HREI m/z calcd. for $\mathrm{C}_{12} \mathrm{H}_{23} \mathrm{OSn}: 299.0766\left({ }^{116} \mathrm{Sn}, \mathrm{M}-\mathrm{C}_{4} \mathrm{H}_{9}\right)^{+}$, found: 299.0770 .

[(3E,5Z)-6-(Tributylstannyl)hepta-3,5-dien-1-ynyl]trimethylsilane (Z-3). A flame dried $1 \mathrm{~L}$ round bottom flask fitted with a heavy Teflon-coated stir bar was cooled under a stream of argon and charged with tri- $n$-butyl-(3-trimethylsilyl-2-propynyl)phosphonium bromide (13,9.25 g, $23.5 \mathrm{mmol})$ and THF $(550 \mathrm{~mL})$. The flask was topped with a rubber septum. While stirring the solution was cooled to between -5 to $0{ }^{\circ} \mathrm{C}$ and treated with sodium bis(trimethylsilyl)amide (1.0 M in THF) (25.8 mL), giving an amber heterogeneous solution. The stirred mixture was warmed to rt over $40 \mathrm{~min}$, then cooled to between -105 to $-99{ }^{\circ} \mathrm{C}$ (internal temperature reading) in a pentane-liquid nitrogen bath. A precooled solution of (Z)-3-tributylstannyl-but-2-en-1-al (7.67 g, $21.3 \mathrm{mmol})$ in THF $(150 \mathrm{~mL})$ was transferred dropwise at a rate of about $1.0 \mathrm{~mL} / \mathrm{min}$ to the phosphonium salt using a precooled cannula, with vigorous stirring and careful temperature regulation (as needed adding portions of liquid nitrogen to the cooling bath). After $2 \mathrm{~h}$ at this cold temp., the work-up was begun by rapid addition of ice-cold propylene oxide $(5.0 \mathrm{~mL})$ followed by trimethylchlorosilane $(5.0 \mathrm{~mL})$. The cooling bath was removed and the solution warmed with stirring over $25 \mathrm{~min}$. It was then poured into a $1 \mathrm{~L}$ separatory funnel containing sat. $\mathrm{NaHCO}_{3}(\mathrm{aq})(150 \mathrm{~mL})$ and extracted using hexanes $(3 \times 140 \mathrm{~mL})$. The combined organic layers were washed using water, and brine, and then dried over anhydrous $\mathrm{Na}_{2} \mathrm{SO}_{4}$. The dried organics were concentrated under reduced pressure leaving a dark oil. The crude product was loaded on silica gel (doped with triethylamine $3 \%, \mathrm{v} / \mathrm{v}$ ) and eluted with hexanes. Purified (3E,5Z)-stannyldienyne $Z-3, \mathrm{R}_{\mathrm{f}}=0.56$ (PMA stain, hexanes) was obtained (10.12 $\mathrm{g}, 96 \%$ ) as a mixture $3 Z: 3 E\left(90: 10\right.$ by ${ }^{1} \mathrm{H}$ NMR) as a yellow oil. ${ }^{1} \mathrm{H}$ NMR $\left(500 \mathrm{MHz}, \mathrm{CDCl}_{3}\right) \delta 6.67$ (app dd, $J=10.9,1.6 \mathrm{~Hz},{ }^{3} J_{\mathrm{SnH}}=58.4 \mathrm{~Hz}$, 1H), 6.51 (app. dd, $J=10.9,15.2 \mathrm{~Hz}, 1 \mathrm{H}), 5.49$ (d, $J=15.2 \mathrm{~Hz}, 1 \mathrm{H}),[5.37$ (d, $J=11 \mathrm{~Hz}, Z$-isomer, 1 
H)], 2.03 (app. d, $J=1.8 \mathrm{~Hz},{ }^{3} J_{\mathrm{SnH}}=23.0 \mathrm{~Hz}, \mathrm{CH}_{3}$ ), $1.49(\mathrm{~m}, 6 \mathrm{H}), 1.32$ (app. sextet, $J=7.2,7.6 \mathrm{~Hz}$, 6H), 0.98 (app. t, $J=8.1 \mathrm{~Hz}, 6 \mathrm{H}), 0.90$ (app. t, $J=7.2 \mathrm{~Hz}, 9 \mathrm{H}), 0.19$ (s, 9H); ${ }^{13} \mathrm{C}$ NMR $(100 \mathrm{MHz}$, $\left.\mathrm{CDCl}_{3}\right) \delta 153.46$ (quat.), $144.49\left({ }^{2} J_{\mathrm{SnC}}=20.2 \mathrm{~Hz}\right), 140.17\left({ }^{3} J_{\mathrm{SnC}}=9.9 \mathrm{~Hz}\right), 109.78,105.18$ (alkyne), 97.00 (alkyne), 29.3, 27.5, $28.02\left(\mathrm{CH}_{3}\right), 13.9,10.4,0.19$ (TMS); MS (m/z, rel \%): 73(60), 121(22), 135(49), 149(52), 193(29), 283(32), 341(22), 397(100); HREI m/z calcd. for $\mathrm{C}_{18} \mathrm{H}_{33} \mathrm{SiSn} 393.1369$ $\left({ }^{116} \mathrm{Sn}, \mathrm{M}-\mathrm{C}_{4} \mathrm{H}_{9}\right)^{+}$, found 393.1378 .

General procedure for Negishi couplings (all of Table 1, and Table 2, products 14-16). Zinc(II) chloride (0.96 g, 7.04 mmol, anhydrous beads) was fused under high vacuum in a Schlenk flask, and cooled under argon. THF $(8 \mathrm{~mL})$ was introduced and the solution was sonicated for $5 \mathrm{~min}$, and then cooled to $-78{ }^{\circ} \mathrm{C}$ under argon. The solution was treated with $n$-BuLi (1.4 mL, $\left.3.70 \mathrm{mmol}\right)$ and then warmed to rt over 25 min. The stannyldienyne $\boldsymbol{E - 2}$ or $\boldsymbol{Z}-3$ (1.59 g, $3.51 \mathrm{mmol})$ was taken up in THF $(2.0 \mathrm{~mL})$, cooled to $-78{ }^{\circ} \mathrm{C}$ under argon, treated with $n$-BuLi $(1,4 \mathrm{~mL}, 3.70 \mathrm{mmol})$ and then stirred for 10 min giving a brown mixture. The lithiated dienyne was transferred to the solution of $n$-butylzinc chloride that had been recooled to $-78{ }^{\circ} \mathrm{C}$, and the combined mixture was stirred at $-78{ }^{\circ} \mathrm{C}$ for 25 min before warming to $\mathrm{rt}$. The vinylic iodide $(3.51 \mathrm{mmol})$ was combined with THF $(3.0 \mathrm{~mL}) \mathrm{plus}$ tetrakis(triphenylphosphine)palladium $\left(0.23 \mathrm{~g}, 1.99 \times 10^{-4} \mathrm{~mol}\right)$ under Argon in a Schlenk flask fitted with a Teflon-coated stir bar. Then, this mixture was added to the in situ prepared diorganozinc species. The combined mixture was degassed by the freeze-pump-thaw method (3x), stirred under Argon at rt for $12 \mathrm{~h}$, then diluted with hexanes $(20 \mathrm{~mL})$ and poured into a separatory funnel containing sat. aqueous $\mathrm{NH}_{4} \mathrm{Cl}(10 \mathrm{~mL})$ and extracted with hexanes $(3 \times 15 \mathrm{~mL})$. The combined organic layers were washed using water, and brine, and then dried over anhydrous $\mathrm{Na}_{2} \mathrm{SO}_{4}$. The dried organics were filtered and concentrated under reduced pressure.

[(3E,5E,7E)-11-(Benzyloxy)-6-methylundeca-3,5,7-trien-1-ynyl]trimethylsilane (Table 1, entry A). The crude product was loaded onto silica gel (doped with triethylamine, $1 \% \mathrm{v} / \mathrm{v}$ ) and eluted with $\mathrm{Et}_{2} \mathrm{O}-$ hexanes 1:20. Purified all-trans-product, $\mathrm{R}_{\mathrm{f}}=0.34$ (Seebach stain, $\mathrm{Et}_{2} \mathrm{O}$-hexanes 1:20), was obtained as 
a yellow oil; 76\%; ${ }^{1} \mathrm{H}$ NMR $\left(500 \mathrm{MHz}, \mathrm{CDCl}_{3}\right) \delta$ 7.39-7.23 (m, Ar-5H), $7.02(\mathrm{dd}, J=15.4,11.7 \mathrm{~Hz}$, $1 \mathrm{H}), 6.12(\mathrm{~d}, J=15.4 \mathrm{~Hz}, 1 \mathrm{H}), 6.02(\mathrm{~d}, J=11.4 \mathrm{~Hz}, 1 \mathrm{H}), 5.82(\mathrm{dt}, J=15.5,7 \mathrm{~Hz}, 1 \mathrm{H}), 5.62(\mathrm{~d}, J=15.4$ $\mathrm{Hz}, 1 \mathrm{H}), 4.52\left(\mathrm{~s}\right.$, benzylic- $\left.\mathrm{CH}_{2}\right), 3.51\left(\mathrm{t}, J=6.5 \mathrm{~Hz}, \mathrm{CH}_{2}\right), 2.27\left(\mathrm{q}, J=7.0 \mathrm{~Hz}, \mathrm{CH}_{2}\right), 1.89\left(\mathrm{~s}, \mathrm{CH}_{3}\right), 1.76$ (app. quint., $J=$ ca. $\left.7.0 \mathrm{~Hz}, \mathrm{CH}_{2}\right), 0.20$ (s, $\left.\mathrm{SiMe}_{3}, 9 \mathrm{H}\right) ;{ }^{13} \mathrm{C} \mathrm{NMR}\left(100 \mathrm{MHz}, \mathrm{CDCl}_{3}\right) \delta 139.49,138.74$; $138.67 ; 134.95 ; 131.7 ; 128.56 ; 128.5 ; 127.84 ; 127.73 ; 110.07 ; 105.59 ; 97.71 ; 73.08 ; 69.79 ; 29.91 ;$ 29.64; 13.15; 0.18; IR (NaCl, neat): 3032, 2956, 2855, 2168, 2119, 1599, 1454, 1102, $845 \mathrm{~cm}^{-1}$; MS (m/z, rel \%) 59(17), 73(100), 91(61), 129(9), 159(7), 203(5), 247(5), 338; HREI m/z calcd. for $\mathrm{C}_{22} \mathrm{H}_{30} \mathrm{OSi}$ : $338.2066(\mathrm{M})^{+}$, found: 338.2076 .

[(3E,5E,7E)-8-(3,4-Bis(benzyloxy)phenyl]-6-methylocta-3,5,7-trien-1-ynyl) trimethylsilane (Table

1, entry B). The crude product was loaded onto silica gel (doped with triethylamine, $1 \% \mathrm{v} / \mathrm{v}$ ) and eluted with hexanes. Purified all-trans-product, $\mathrm{R}_{\mathrm{f}}=0.21$ (PMA stain, $\mathrm{Et}_{2} \mathrm{O}$ :hexanes 1:10), was obtained as a yellow solid; $\mathrm{mp}=100-102{ }^{\circ} \mathrm{C} ; 61 \%{ }^{1} \mathrm{H} \mathrm{NMR}\left(400 \mathrm{MHz}, \mathrm{CDCl}_{3}\right) \delta$ 7.49-7.31 (complex m, Ar-10H), 7.07 (app. d, $J=2.0 \mathrm{~Hz}, \mathrm{Ar}-\mathrm{H}), 7.06$ (app. dd, $J=11.6,15.2 \mathrm{~Hz}$, vinyl-H), 6.95 (dd, $J=2.0,8.4 \mathrm{~Hz}, \mathrm{Ar}-$ H), $6.89(\mathrm{~d}, J=8.4 \mathrm{~Hz}, \mathrm{Ar}-\mathrm{H}), 6.66(\mathrm{~d}, J=16.0 \mathrm{~Hz}$, vinyl-H), 6.55 (d, $J=16.0 \mathrm{~Hz}$, vinyl-H), $6.21(\mathrm{~d}, J=$ $11 \mathrm{~Hz}, 1 \mathrm{H}), 5.67(\mathrm{~d}, J=16 \mathrm{~Hz}, 1 \mathrm{H}), 5.18\left(\mathrm{~s}\right.$, benzylic- $\left.\mathrm{CH}_{2}\right), 5.17\left(\mathrm{~s}\right.$, benzylic- $\left.\mathrm{CH}_{2}\right), 2.0\left(\mathrm{~s}, \mathrm{CH}_{3}\right), 0.21$ (s, TMS-9H); ${ }^{13} \mathrm{C}$ NMR $\left(100 \mathrm{MHz}, \mathrm{CDCl}_{3}\right) \delta 149.3,149.1,139.4,138.7,137.4,137.3,131.6,131.4$, $130.5,129.3,128.7,128.1,128.0,127.6,127.4,120.8,115.1,112.9,110.7,105.6,98.5,71.6,71.4,13.1$ 0.18; IR (KBr) 3034, 2959, 2870, 2170, 2121, 1576, 1506, 1455, 1387, 1262, 1134, 1004, 953, 846, 743, 697; MS (m/z, rel \%): 73(17), 91(100), 179(2), 387(2), 478(17); HREI m/z calcd. for $\mathrm{C}_{32} \mathrm{H}_{34} \mathrm{O}_{2} \mathrm{Si}_{\text {: }}$ $478.2328(\mathrm{M})^{+}$, found: 478.2334 .

\section{[(3E,5E)-6-(1,2,3,4-Tetrahydro-1,1,4,4-tetramethyl-6-propoxynaphthalen-7-yl)hepta-3,5-dien-1-}

ynyl]trimethylsilane (Table 1, entry C). The crude product was loaded on silica gel (doped with triethylamine, $1 \% \mathrm{v} / \mathrm{v}$ ) and eluted with EtOAc:hexanes 1:50. All-trans-dienyne product, $\mathrm{R}_{\mathrm{f}}=0.35$ (vanillin stain, EtOAc:hexanes 1:50), was obtained as a yellow residue containing some recovered stannyldienyne $\boldsymbol{E}-2$ plus triphenylphosphine, based on the ${ }^{1} \mathrm{H}$ NMR spectrum. A second chromatography 
was partially successful in purifing this product. Comparison with starting material NMR and mass spectra allowed the following tentative assignment for somewhat impure all-trans-dienyne; $53 \% ;{ }^{1} \mathrm{H}$ NMR (400 MHz, $\left.\mathrm{CDCl}_{3}\right) \delta 7.06(\mathrm{~s}, \mathrm{Ar}-\mathrm{H}), 7.05(\mathrm{dd}, J=11.5,15.6 \mathrm{~Hz}$, vinyl-H), 6.74 (s, Ar-H), 6.22 (app. dd, $J=0.8,11.4 \mathrm{~Hz}$, vinyl-H-5), $5.62\left(\mathrm{~d}, J=15.3 \mathrm{~Hz}\right.$, vinyl-H), 3.88 (t, $\left.J=6.3 \mathrm{~Hz}, \mathrm{CH}_{2}-\mathrm{O}\right), 2.18$ (d, $1.2 \mathrm{~Hz}, \mathrm{CH}_{3}$ ), 1.78 (sextet, $\left.J=7.2 \mathrm{~Hz}, \mathrm{CH}_{2}\right), 1.66$ (app. br. s, $\left.2 \mathrm{CH}_{2}\right), 1.07\left(\mathrm{~s}, 2 \mathrm{CH}_{3}\right), 1.05\left(\mathrm{~s}, 2 \mathrm{CH}_{3}\right)$, $0.95\left(\mathrm{t}, J=7.5 \mathrm{~Hz}, \mathrm{CH}_{3}\right), 0.21(\mathrm{~s}, \mathrm{TMS}) ;{ }^{13} \mathrm{C} \mathrm{NMR}\left(100 \mathrm{MHz}, \mathrm{CDCl}_{3}\right) \delta 154.3,145.5,141.1,140.5$, $140.0,137.4,131.8,128.1,127.3,112.0,110.0,105.7,89.1,87.4,70.0,35.6,32.0,22.0,18.1,14.3,11.0$ 0.1; MS (m/z, rel \%): 73(53), 111(12), 215(18), 255(6), 365(7), 393(52), 408(100); HREI m/z calcd. for $\mathrm{C}_{27} \mathrm{H}_{40} \mathrm{OSi}: 408.2848(\mathrm{M})^{+}$, found: 408.2837.

\section{1,3-Dimethyl-5-((2E,4E)-7-(trimethylsilyl)hepta-2,4-dien-6-yn-2-yl)pyrimidine-2,4 (1H,3H)-dione}

(Table 1, entry D). The crude product was loaded on silica gel (doped with triethylamine, 1\% v/v) and eluted with hexanes. Purified product, $\mathrm{R}_{\mathrm{f}}=0.19$ (PMA stain, EtOAc-hexanes 1:3), was obtained as a yellow solid; $\mathrm{mp}=110-112{ }^{\circ} \mathrm{C} ; 41 \% ;{ }^{1} \mathrm{H} \mathrm{NMR}\left(400 \mathrm{MHz}, \mathrm{CDCl}_{3}\right) \delta 7.17$ (s, uracil vinyl-H), 6.99 (dd, $J=11.9,15.5 \mathrm{~Hz}$, vinyl-H), 6.97 (app. dt, $J=1.7,11.5 \mathrm{~Hz}, 1 \mathrm{H}), 5.95$ (d, $J=15.4 \mathrm{~Hz}, 1 \mathrm{H}), 3.44$ (s, $\left.\operatorname{uracil}-\mathrm{NCH}_{3}\right), 3.36\left(\mathrm{~s}\right.$, uracil- $\left.\mathrm{NCH}_{3}\right), 2.07\left(\mathrm{~d}, J=1.0 \mathrm{~Hz}, \mathrm{CH}_{3}\right), 0.21(\mathrm{~s}, \mathrm{TMS}-9 \mathrm{H}) ;{ }^{13} \mathrm{C}$ NMR $(100$ $\left.\mathrm{MHz}, \mathrm{CDCl}_{3}\right) \delta 162.3,151.4,139.6,138.9,132.9,128.6,116.3,112.1,105.1,98.3,37.4,28.3,16.4$, 0.15; IR (KBr) 3344, 3295, 3084, 3044, 2959, 2900, 2159, 2114, 1705, 1653, 1592, 1450, 1358, 1236, 1122, 1064, 1028, 963, 850, 752, 657, $527 \mathrm{~cm}^{-1}$; LREIMS m/z: $302.287(\mathrm{M})^{+}$; HREI m/z calcd. for $\mathrm{C}_{15} \mathrm{H}_{19} \mathrm{~N}_{2} \mathrm{O}_{2} \mathrm{Si}: 287.1216\left(\mathrm{M}-\mathrm{CH}_{3}\right)^{+}$, found: 287.1209 .

Trimethyl[(3E,5E,7E)-6-methyltetradeca-3,5,7-trien-1-ynyl]silane (Table 1, entry E). The crude product was loaded on silica gel (doped with triethylamine, 1\% v/v) and eluted with hexanes. Purified all-trans-product, $\mathrm{R}_{\mathrm{f}}=0.31$ (PMA stain, hexanes), was obtained as a yellow oil; 91\%; ${ }^{1} \mathrm{H}$ NMR (400 $\left.\mathrm{MHz}, \mathrm{CDCl}_{3}\right) \delta 7.02(\mathrm{dd}, J=11.6,15.4,1 \mathrm{H}), 6.09$ (app. d, $\left.J=15.5 \mathrm{~Hz}, 1 \mathrm{H}\right), 6.01$ (app. d, $J=11.8$ $\mathrm{Hz}, 1 \mathrm{H}), 5.82$ (app. dt, $J=7.5,15.2 \mathrm{~Hz}, 1 \mathrm{H}), 5.59$ (d, $J=15.3 \mathrm{~Hz}, 1 \mathrm{H}), 2.14$ (q, $\left.J=7.1 \mathrm{~Hz}, \mathrm{CH}_{2}\right), 1.89$ (br. s, $\left.\mathrm{CH}_{3}\right), 1.41(\mathrm{~m}, 2 \mathrm{H}), 1.29(\mathrm{~m}, 6 \mathrm{H}), 0.89$ (app. t, $\left.J=6.5 \mathrm{~Hz}, \mathrm{CH}_{3}\right), 0.19(\mathrm{~s}, 9 \mathrm{H}) ;{ }^{13} \mathrm{C}$ NMR $(125$ 
$\left.\mathrm{MHz}, \mathrm{CDCl}_{3}\right) \delta 139.6,138.9,134.4,132.8,128.3,109.9,105.7,97.6,33.4,31.9,29.6,29.2,22.8,14.3$, 13.2, 0.21; MS (m/z, rel \%): 59(28), 73(100), 105(5), 149(11), 175(7), 189(38), 203(6), 259(6), 274(18). HREI m/z calcd. for $\mathrm{C}_{18} \mathrm{H}_{30} \mathrm{Si}: 274.2119(\mathrm{M})^{+}$, found: 274.2116 .

[(3E,5E,7E)-Trimethyl-6-methyl-8-phenylocta-3,5,7-trien-1-ynyl]silane (Table 1, entry F). Since $\beta$ bromostyrene was used as a cis/trans mixture of isomers (Acros 16929 0250), the crude coupling product was obtained as a 7-E:Z mixture (9:1) that was separated on a silica gel column (doped with triethylamine, $1 \% \mathrm{v} / \mathrm{v}$ ) and eluted with EtOAc:hexanes 1:25. Purified all-trans-product, $\mathrm{R}_{\mathrm{f}}=0.22(\mathrm{PMA}$ stain, $\mathrm{Et}_{2} \mathrm{O}$ :hexanes 1:45), was obtained as a yellow solid; $66 \% ;{ }^{1} \mathrm{H} \mathrm{NMR}\left(400 \mathrm{MHz}, \mathrm{CDCl}_{3}\right) \delta 7.44(\mathrm{~d}, \mathrm{~J}$ $=7.4 \mathrm{~Hz}, 1 \mathrm{H}), 7.36-7.22(\mathrm{~m}, \mathrm{Ar}-4 \mathrm{H}), 7.06(\mathrm{dd}, J=11.6,15.6 \mathrm{~Hz}, 1 \mathrm{H}), 6.85(\mathrm{~d}, J=16.0 \mathrm{~Hz}, 1 \mathrm{H}), 6.67$ (d, $J=16.1 \mathrm{~Hz}, 1 \mathrm{H}), 6.26(\mathrm{~d}, J=11.6 \mathrm{~Hz}, 1 \mathrm{H}), 5.70(\mathrm{~d} ., J=15.2 \mathrm{~Hz}, 1 \mathrm{H}), 2.04\left(\mathrm{~s}, \mathrm{CH}_{3}\right), 0.22(\mathrm{~s}, \mathrm{TMS})$; ${ }^{13} \mathrm{C}$ NMR $\left(125 \mathrm{MHz}, \mathrm{CDCl}_{3}\right) \delta 139.3,138.6,137.5,133.1,131.2,129.7,128.9,127.8,126.7,111.2$, 105.5, 98.7, 13.1, 0.18; MS (m/z, rel \%): 59(28), 73(100), 91(12), 135(8), 155(11), 193(11), 251 (14), 266(44); HREI m/z calcd. for $\mathrm{C}_{18} \mathrm{H}_{22} \mathrm{Si}: 266.1491(\mathrm{M})^{+}$, found: 266.1490 .

\section{4-[(1E,3E,5E)-3-Methyl-8-(trimethylsilyl)octa-1,3,5-trien-7-ynyl]phenyl-4-methyl-benzenesul-}

fonate (Table 1, entry G). The mixture was stirred under argon at $\mathrm{rt}$ for $3 \mathrm{~h}$, then diluted with $\mathrm{Et}_{2} \mathrm{O}$ (15 $\mathrm{mL})$ and silica gel $(2 \mathrm{~g})$ was added. The volatiles were stripped and the residue was loaded on silica gel (doped with triethylamine, $1 \% \mathrm{v} / \mathrm{v}$ ) and eluted with $\mathrm{Et}_{2} \mathrm{O}$-hexanes 1:7. Purified product, $\mathrm{R}_{\mathrm{f}}=0.32$ (PMA stain, $\mathrm{Et}_{2} \mathrm{O}$-hexanes 1:10), was obtained as a mixture of isomers as a solid. (The product was unstable at $\mathrm{rt}$ in air and discolored from yellow to tan over several hours. The product had moderate stability when frozen in degassed PhH.); 81\%; ${ }^{1} \mathrm{H}$ NMR (400 MHz, $\left.\mathrm{CDCl}_{3}\right) \delta 7.61$ (d, J=8.3 Hz, Ar2H), [7.46 (d, $J=8.3 \mathrm{~Hz})], 7.22(\mathrm{~d}, J=8.8 \mathrm{~Hz}, \mathrm{Ar}-2 \mathrm{H}), 7.21(\mathrm{~d}, J=8.4 \mathrm{~Hz}, \mathrm{Ar}-2 \mathrm{H}), 7.16$ (s, residual $\mathrm{PhH}$ ), [7.11 (d, $J=8.0 \mathrm{~Hz}$ )], $6.93(\mathrm{dd}, J=11.7,15.4 \mathrm{~Hz}$, vinyl-H), 8.67 (app. d, $J=8.7 \mathrm{~Hz}$, tosyl-Ar2H), [6.73 (d, $J=15.2 \mathrm{~Hz}], 6.67(\mathrm{~d}, 15.9 \mathrm{~Hz}, 1 \mathrm{H}),[6.51(\mathrm{~d}, J=7.9 \mathrm{~Hz})], 6.47(\mathrm{~d}, J=15.9 \mathrm{~Hz}, 1 \mathrm{H}), 6.14$ (d, $J=15.8 \mathrm{~Hz}$, vinyl-H), 5.59 (d, $J=15.3 \mathrm{~Hz}$, vinyl-H), 2.35 (s, tosyl- $\mathrm{CH}_{3}$ ), 1.89 (s, $\left.\mathrm{C}_{3}-\mathrm{CH}_{3}\right), 0.12$ (s, TMS-9H); HREI m/z calcd. for $\mathrm{C}_{26} \mathrm{H}_{28} \mathrm{O}_{3} \mathrm{SiS} 436.1528(\mathrm{M})^{+}$, found 436.1533 . 
t-Butyldiphenylsilyl protected 3,5,5-trimethyl-4-((3E,5E)-3-methyl-8-(trimethylsilyl)octa-3,5-dien-

1,7-diynyl)cyclohex-3-enol (Table 1, entry H). The crude product was loaded on silica gel (doped with triethylamine, $3 \% \mathrm{v} / \mathrm{v}$ ) and eluted with $\mathrm{Et}_{2} \mathrm{O}$-hexanes 1:99. Purified coupling product, $\mathrm{R}_{\mathrm{f}}=0.35$ (PMA stain), Et $2 \mathrm{O}$-hexanes 1:50, was obtained as a yellow oil; 80\%; ${ }^{1} \mathrm{H}-\mathrm{NMR}\left(400 \mathrm{MHz}, \mathrm{CDCl}_{3}\right)$ 7.71-7.63 (m, Ar-4H), 7.44-7.33 (m, Ar-6H), $6.68(\mathrm{dd}, J=11.8,15.4 \mathrm{~Hz}$, vinyl-H-5), 6.32 (dd, $J=0.8$, 11.7 Hz, vinyl-H-4), 5.61 (d, $J=15.3 \mathrm{~Hz}$, vinyl-H-6), 3.92 (m, methine-H), 2.22 (m, 2H), 1.95 (d, $J=$ $\left.1.0 \mathrm{~Hz}, \mathrm{C} 3-\mathrm{CH}_{3}\right), 1.82\left(\mathrm{~s}, \mathrm{CH}_{3}\right), 1.64(\mathrm{dd}, J=3.2,12.6 \mathrm{~Hz}, 1 \mathrm{H}), 1.50(\mathrm{t}, J=12.4 \mathrm{~Hz}, 1 \mathrm{H}), 1.08(\mathrm{~s}$, tert$\mathrm{Bu}, 9 \mathrm{H}), 1.09\left(\mathrm{~s}, \mathrm{CH}_{3}\right), 1.08$ (s, tert-Bu, 9H), $0.78\left(\mathrm{~s}, \mathrm{CH}_{3}\right), 0.21$ (s, TMS-9H); ${ }^{13} \mathrm{C}-\mathrm{NMR}(100 \mathrm{MHz}$, $\left.\mathrm{CDCl}_{3}\right)_{-} 139.3,138.4,136.0,135.9,134.6,134.5,133.2,129.8,127.8,123.9,123.0,111.1,105.2$, 98.6, 97.6, 90.3, 66.4, 46.7, 41.9, 36.6, 30.6, 28.4, 27.2, 22.7, 19.3, 18.4, 0.14; HREI m/z calcd. for $\mathrm{C}_{37} \mathrm{H}_{48} \mathrm{OSi} 564.3244(\mathrm{M})^{+}$, found 564.3253.

Trimethyl((3E,5Z,7E)-6-methyltetradeca-3,5,7-trien-1-ynyl)silane (14). The crude product was loaded on silca gel (doped with triethylamine, $1 \% \mathrm{v} / \mathrm{v}$ ) and eluted with hexanes. Purified (5Z)-trienyne 14, $\mathrm{R}_{\mathrm{f}}=0.49$ (PMA stain), hexanes, was obtained as a yellow oil; $81 \%$ (mixture $3 E: 3 Z=90: 10$ determined by ${ }^{1} \mathrm{H}$ NMR); ${ }^{1} \mathrm{H}$ NMR $\left(400 \mathrm{MHz}, \mathrm{CDCl}_{3}\right) \delta 7.13(\mathrm{dd}, J=11.6,15.4 \mathrm{~Hz}$, vinyl), $6.62(\mathrm{~d}, J$ = 15.6 Hz, vinyl-H), 5.89 (d, $J=11.7 \mathrm{~Hz}$, vinyl-H), 5.81 (app. quint., $J=$ ca. $7.0 \mathrm{~Hz}$, vinyl-H), 5.52 (d, $J=15.3 \mathrm{~Hz}$, vinyl-H-3), 2.16 (q, $J=7.0 \mathrm{~Hz}, 2 \mathrm{H}), 1.89$ (br. s, $\left.\mathrm{CH}_{3}\right), 1.42-1.37$ (m, 2H), 1.33-1.28 (m, $6 \mathrm{H}), 0.89\left(\mathrm{~m}, \mathrm{CH}_{3}\right), 0.20$ (s, TMS-9H); ${ }^{13} \mathrm{C} \mathrm{NMR}\left(100 \mathrm{MHz}, \mathrm{CDCl}_{3}\right) \delta 143.4,138.4,137.7,134.1$, 126.8, 109.2, 105.6, 97.1, 33.6, 31.9, 29.6, 29.2, 22.8, 21.1, 14.3, 0.19; MS (m/z, rel \%): 59(27), 83(7), 93(4), 149(11), 175(6), 189(38), 203(7), 259(7), 274(22); HREI m/z calcd. for $\mathrm{C}_{18} \mathrm{H}_{30} \mathrm{Si}: 274.2117$ $(\mathrm{M})^{+}$, found: 274.2111 .

Trimethyl[(3E,5Z)-6-(3,5-dimethylphenyl)hepta-3,5-dien-1-ynyl]silane (15). The crude product was loaded on silca gel (doped with triethylamine, $2 \% \mathrm{v} / \mathrm{v}$ ) and eluted with hexanes. Purified 5-(Z)-trienyne 15, $\mathrm{R}_{\mathrm{f}}=0.18$ (PMA stain), was obtained as an oil; 88\%; ${ }^{1} \mathrm{H}$ NMR $\left(400 \mathrm{MHz}, \mathrm{CDCl}_{3}\right) \delta 6.95(\mathrm{bs}, 1 \mathrm{H})$; $6.83(\mathrm{~s}, 2 \mathrm{H}) ; 6.68(\mathrm{dd}, \mathrm{J}=11.2,15.6 \mathrm{~Hz}, 1 \mathrm{H}) ; 6.14(\mathrm{~d}, \mathrm{~J}=11.2 \mathrm{~Hz}, 1 \mathrm{H}) ; 5.59(\mathrm{~d}, \mathrm{~J}=15.6 \mathrm{~Hz}, 1 \mathrm{H}) ; 2.34$ 
$(\mathrm{s}, 3 \mathrm{H}) ; 2.34(\mathrm{~s}, 3 \mathrm{H}) ; 2.12(\mathrm{~s}, 3 \mathrm{H}) ; 0.64(\mathrm{~s}, 9 \mathrm{H})$; HREI m/z calcd. for $\mathrm{C}_{18} \mathrm{H}_{24} \mathrm{Si}: 268.1647$ (M) ${ }^{+}$; found: 268.1652.

[(3E,5Z,7E)-11-(Benzyloxy)-6-methylundeca-3,5,7-trien-1-ynyl]trimethylsilane (16). The crude product was loaded on silca gel (doped with triethylamine, 1\% v/v) and eluted with hexanes:Et ${ }_{2} \mathrm{O}$ 20:1. Purified 5-(Z)-trienyne $16, \mathrm{R}_{\mathrm{f}}=0.34$, was obtained as a yellow oil; 55\%; ${ }^{1} \mathrm{H}$ NMR $\left(400 \mathrm{MHz}, \mathrm{CDCl}_{3}\right) \delta$ 7.37-7.27 (m, 5H), $7.12(\mathrm{dd}, J=12 \mathrm{~Hz}, 15.6 \mathrm{~Hz}, 1 \mathrm{H}), 6.62(\mathrm{~d}, J=16 \mathrm{~Hz}, 1 \mathrm{H}), 5.9(\mathrm{~d}, J=12 \mathrm{~Hz}, 1 \mathrm{H})$, $5.81(\mathrm{dt}, J=15.6,7.2 \mathrm{~Hz}, 1 \mathrm{H}), 5.53(\mathrm{~d}, J=15.2 \mathrm{~Hz}, 1 \mathrm{H}), 4.52(\mathrm{~s}, 2 \mathrm{H}), 3.50(\mathrm{t}, J=6.4 \mathrm{~Hz}, 2 \mathrm{H}), 2.27(\mathrm{~m}$ $2 \mathrm{H}), 1.89$ (s, $3 \mathrm{H}), 1.76$ (app. quint, 2H), $0.21(\mathrm{~s}, 9 \mathrm{H}) ;{ }^{13} \mathrm{C} \mathrm{NMR}\left(100 \mathrm{MHz}, \mathrm{CDCl}_{3}\right) \delta 138.7,138.3$, $137.45,133.0,128.6,127.9,127.8,127.3,127.0,109.4,105.5,97.1,73.1,69.8,30.2,29.6 .21 .0,0.2 ;$ IR (NaCl, neat) 2955, 2926, 2854, 1719, 1594, 1455, 1103, 735, $697 \mathrm{~cm}^{-1} ; \mathrm{MS}$ (m/z, rel \%): 59 (18), 73(100), 105(6), 129(10), 149(14), 159(7), 173(3), 203(4), 232(3), 247(5), 338(5). HRMS m/z calcd. for $\mathrm{C}_{22} \mathrm{H}_{30} \mathrm{Si}: 338.2066(\mathrm{M})^{+}$, found: 338.2068 .

General procedure for Negishi couplings of $Z$-3 (Table 2, products 15, 17-23). Zinc(II) chloride (0.273 g, $2.0 \mathrm{mmol}$, anydrous beads) was fused under high vacuum in a oven dried flask, and cooled under argon. THF (6 mL) was introduced and the solution was slightly warmed to effect dissolution, and then cooled to $\mathrm{rt}$ under argon. The stannyldienyne $\boldsymbol{Z - 3}(0.680 \mathrm{~g}, 1.5 \mathrm{mmol})$ was taken up in THF (12.0 $\mathrm{mL})$, cooled to $-78{ }^{\circ} \mathrm{C}$ under argon, treated with $n$-BuLi $(0.62 \mathrm{~mL}, 1.5 \mathrm{mmol})$ and then stirred for $10 \mathrm{~min}$ giving a green mixture. The solution of zinc chloride was transferred to the lithiated dienyne at $-78^{\circ} \mathrm{C}$, and the combined mixture was stirred at $-78{ }^{\circ} \mathrm{C}$ for $10 \mathrm{~min}$ before warming to rt. The vinylic iodide $(1.00 \mathrm{mmol})$ was combined with THF $(6.0 \mathrm{~mL})$ plus tetrakis(triphenylphosphine)palladium $(0.116 \mathrm{~g}$, $0.10 \mathrm{mmol}$ ) under Argon in a flask fitted with a Teflon-coated stir bar and stirred for $10 \mathrm{~min}$. Then, this mixture was added to the in situ prepared organozinc species. The combined mixture was stirred under Argon at $\mathrm{rt}$ for $12 \mathrm{~h}$, then diluted with hexanes $(20 \mathrm{~mL})$ and poured into a separatory funnel containing sat. aqueous $\mathrm{NH}_{4} \mathrm{Cl}(10 \mathrm{~mL})$ and extracted with hexanes $(3 \times 15 \mathrm{~mL})$. The combined organic layers were 
washed using water, and brine, and then dried over anhydrous $\mathrm{Na}_{2} \mathrm{SO}_{4}$. The dried organics were filtered and concentrated under reduced pressure.

\section{1-(S)-3,5,5-Trimethyl-4-[(1E,3Z,5E)-3-methyl-8-(trimethylsilyl)octa-1,3,5-trien-7-ynyl)cyclohex-3-}

enyloxy)(tert-butyl)(phenyl)silyl]benzene (17). The crude product was loaded on silca gel (doped with triethylamine, 2\% v/v) and eluted with hexanes: EtOAc 80:1. Purified (5Z)-trienyne 17, $\mathrm{R}_{\mathrm{f}}=0.28$ (PMA stain), was obtained as an oil; 62\%; ${ }^{1} \mathrm{H}$ NMR $\left(300 \mathrm{MHz}, \mathrm{CDCl}_{3}\right) \delta 7.71(\mathrm{~m}, 4 \mathrm{H}) ; 7.40(\mathrm{~m}, 6 \mathrm{H}) ; 7.06(\mathrm{dd}$, $\mathrm{J}=11.7,15.4 \mathrm{~Hz}, 1 \mathrm{H}) ; 6.52(\mathrm{~d}, \mathrm{~J}=16.1 \mathrm{~Hz}, 1 \mathrm{H}) ; 6.11(\mathrm{~d}, \mathrm{~J}=16.1 \mathrm{~Hz}, 1 \mathrm{H}) ; 5.95(\mathrm{~d}, \mathrm{~J}=11.4 \mathrm{~Hz}, 1 \mathrm{H})$; $5.54(\mathrm{~d}, \mathrm{~J}=15.4 \mathrm{~Hz}, 1 \mathrm{H}) ; 3.95(\mathrm{~m}, 1 \mathrm{H}) ; 2.22(\mathrm{~m}, 2 \mathrm{H}) ; 1.92(\mathrm{~s}, 3 \mathrm{H}) ; 1.66(\mathrm{~s}, 3 \mathrm{H}) ; 1.57(\mathrm{~m}, 2 \mathrm{H}) ; 1.09(\mathrm{~s}$, 9H); 0.69 (s, 3H); $0.20(\mathrm{~s}, 9 \mathrm{H}) ;{ }^{13} \mathrm{C} \mathrm{NMR}\left(75 \mathrm{MHz}, \mathrm{CDCl}_{3}\right) \delta$ 138.2, 137.7, 137.6, 136.1, 136.0, 134.8, $130.1,129.7,129.4,128.8,127.9,127.7,127.6,109.6,105.5,97.6,77.4,66.7,48.4,42.9,37.0,30.3$, $28.3,27.2,21.8,20.8,19.3,0.20$.

[(3E,5Z)-6-(1,2,3,4-Tetrahydro-1,1,4,4-tetramethyl-6-propoxynaphthalen-7-yl)hepta-3,5-dien-1ynyl]trimethylsilane (18). The crude product was loaded on silica gel (doped with triethylamine, $2 \%$ $\mathrm{v} / \mathrm{v}$ ) and eluted with hexanes:EtOAc 80:1. Purified 5-(Z)-trienyne 18, $\mathrm{R}_{\mathrm{f}}=0.24$ (PMA stain), was obtained as a yellow oil; 94\%; ${ }^{1} \mathrm{H}$ NMR $\left(300 \mathrm{MHz}, \mathrm{CDCl}_{3}\right) \delta 6.94$ (bs, 1H); 6.77 (bs, 1H); 6.59 (dd, J = 11.4, 15.8 Hz, 1H); $6.15(\mathrm{~d}, \mathrm{~J}=11.1 \mathrm{~Hz}, 1 \mathrm{H}) ; 5.53(\mathrm{~d}, \mathrm{~J}=15.5 \mathrm{~Hz}, 1 \mathrm{H}) ; 3.88(\mathrm{t}, \mathrm{J}=6.5 \mathrm{~Hz}, 2 \mathrm{H}), 2.14$ (bs, 3H); 1.76, (sextet, J = 6.6 Hz, 2H); 1.69 (bs, 2H); 1.31 (s, 6H); 1.26 (s, 6H); 1.00 (t, J = 7.2 Hz, 3H); 0.15 (s, 9H); ${ }^{13} \mathrm{C}$ NMR $\left(75 \mathrm{MHz}, \mathrm{CDCl}_{3}\right) \delta 154.1,145.4,142.6,141.9,136.6,129.1,127.6,127.4$, 109.9, 108.4, 105.8, 96.0, 70.0, 35.4, 34.7, 33.8, 32.0, 31.8, 25.1, 22.9, 14.3, 11.0, 0.18; HREI m/z calcd. for $\mathrm{C}_{27} \mathrm{H}_{40} \mathrm{OSi}$ : $408.2848(\mathrm{M})^{+}$; found: 408.2860 .

[(3E,5Z,7E)-8-(3,4-Bis(benzyloxy)phenyl]-6-methylocta-3,5,7-trien-1-ynyl) trimethylsilane (19).

The crude product was loaded on silca gel (doped with triethylamine, 1\% v/v) and eluted with EtOAc:hexanes 1:20. Purified 5-(Z)-19 $\mathrm{R}_{\mathrm{f}}=0.28$ (iodine stain, EtOAc:hexanes 1:20) was obtained as a yellow solid but based on the ${ }^{1} \mathrm{H}$ NMR spectrum included small amounts of other isomers; $86 \% ;{ }^{1} \mathrm{H}$ NMR (400 MHz, $\left.\mathrm{CDCl}_{3}\right) \delta 7.51$ (app. d, $J=7.2 \mathrm{~Hz}, \mathrm{Ar}-2 \mathrm{H}$ ), 7.47-7.41 (m, Ar-4H), 7.41-7.29 (complex 
mult., Ar-4H), 7.16 (dd, $J=11.7,15.2 \mathrm{~Hz}$, vinyl-H-5), 7.08 (d, $J=2.0 \mathrm{~Hz}, \mathrm{Ar}-\mathrm{H}), 7.07$ (d, $J=15.6 \mathrm{~Hz}$, vinyl-H), 6.92 (dd, $J=1.8,8.4 \mathrm{~Hz}, \mathrm{Ar}-\mathrm{H}), 6.86$ (d, $J=8.0 \mathrm{~Hz}, \mathrm{Ar}-\mathrm{H}), 6.52$ (d, $J=16 \mathrm{~Hz}$, vinyl-H), 6.02 (d, $J=11.6 \mathrm{~Hz},(Z)$-vinyl-H), 5.60 (d, $J=15.6 \mathrm{~Hz}$, vinyl-H), $5.23\left(\mathrm{~s}, \mathrm{CH}_{2}\right), 5.19\left(\mathrm{~s}, \mathrm{CH}_{2}\right), 1.96\left(\mathrm{~s}, \mathrm{CH}_{3}\right)$, 0.23 (s, TMS); ${ }^{13} \mathrm{C}$ NMR (100 MHz, $\left.\mathrm{CDCl}_{3}\right) \delta$ 149.23, 149.16, 137.95, 137.58, 137.32, 137.28, 131.2, $130.3,128.82,128.80,128.67,128.11,128.0,127.5,127.4,123.6,121.2,114.8,112.9,110.0,105.6$ 98.0, 71.7, 71.3; HREI m/z calcd. for $\mathrm{C}_{32} \mathrm{H}_{34} \mathrm{O}_{2} \mathrm{Si}: 478.2328(\mathrm{M})^{+}$, found: 478.2333 .

Trimethyl[(3E,5Z,7E)-6-methyl-8-(2,6-dimethylcyclohex-1-enyl)octa-3,5,7-trien-1-ynyl]silane (20).

The crude product was loaded on silca gel (doped with triethylamine, $2 \% \mathrm{v} / \mathrm{v}$ ) and eluted with hexanes. Purified 5-(Z)-trienyne 20, $\mathrm{R}_{\mathrm{f}}=0.25$ (PMA stain), was obtained as an oil; $90 \% ;{ }^{1} \mathrm{H}$ NMR $(300 \mathrm{MHz}$, $\left.\mathrm{CDCl}_{3}\right) \delta 7.16(\mathrm{dd}, \mathrm{J}=11.7,15.3 \mathrm{~Hz}, 1 \mathrm{H}) ; 6.78(\mathrm{~d}, \mathrm{~J}=15.9 \mathrm{~Hz}, 1 \mathrm{H}) ; 6.69(\mathrm{~d}, \mathrm{~J}=15.9 \mathrm{~Hz}, 1 \mathrm{H}) ; 5.98(\mathrm{~d}$, $\mathrm{J}=11.1 \mathrm{~Hz}, 1 \mathrm{H}) ; 5.56(\mathrm{~d}, \mathrm{~J}=15.6 \mathrm{~Hz}, 1 \mathrm{H}) ; 2.73(\mathrm{~m}, 1 \mathrm{H}) ; 2.11(\mathrm{~m}, 2 \mathrm{H}) ; 1.99(\mathrm{~s}, 3 \mathrm{H}) ; 1.81(\mathrm{~s}, 3 \mathrm{H}) ; 1.63$ (m, 4H); $0.22(\mathrm{~s}, 9 \mathrm{H}) ;{ }^{13} \mathrm{C}$ NMR $\left(75 \mathrm{MHz}, \mathrm{CDCl}_{3}\right) \delta 138.6,138.3,135.6,133.9,129.0,127.7,122.2$, 109.2, 105.7, 97.6, 33.7, 30.2, 25.2, 21.1, 20.5, 20.0, 17.7, 0.24; HREI m/z calcd for $\mathrm{C}_{20} \mathrm{H}_{30} \mathrm{Si}$ : 298.2117(M) ${ }^{+}$; found: 298.2107.

$[(3 E, 5 Z, 7 E)-8-(B e n z o[d][1,3]$ dioxol-6-yl)-6-methylocta-3,5,7-trien-1-ynyl] trimethylsilane (21). The crude product was loaded on silca gel (doped with triethylamine, $2 \% \mathrm{v} / \mathrm{v}$ ) and eluted with hexanes: EtOAc 25:1. Purified 5-(Z)-trienyne 21, $\mathrm{R}_{\mathrm{f}}=0.31$ (PMA stain), was obtained as an oil; 77\%; ${ }^{1} \mathrm{H}$ NMR $\left(300 \mathrm{MHz}, \mathrm{CDCl}_{3}\right) \delta 7.24(\mathrm{dd}, \mathrm{J}=11.7,15.4 \mathrm{~Hz}, 1 \mathrm{H}) ; 7.21(\mathrm{~d}, \mathrm{~J}=15.8 \mathrm{~Hz}, 1 \mathrm{H}) ; 7.05(\mathrm{~d}, \mathrm{~J}=1.8 \mathrm{~Hz}$, 1H); $6.91(\mathrm{dd}, \mathrm{J}=1.8,8.1 \mathrm{~Hz}, 1 \mathrm{H}) ; 6.79(\mathrm{~d}, \mathrm{~J}=8.1 \mathrm{~Hz}, 1 \mathrm{H}) ; 6.58(\mathrm{~d}, \mathrm{~J}=16.1 \mathrm{~Hz}, 1 \mathrm{H}) ; 6.05(\mathrm{~d}, \mathrm{~J}=11.7$ $\mathrm{Hz}, 1 \mathrm{H}) ; 5.99$ (s, 2H); 5.60 (d, J = 15.0 Hz, 1H); 2.02 (s, 3H); 0.23 (s, 9H); ${ }^{13} \mathrm{C}$ NMR (75 MHz, $\mathrm{CDCl}_{3}$ ) $\delta 148.4,147.8,138.0,137.2,132.1,130.4,128.9,123.6,122.1,110.1,108.7,105.8,105.5,101.4,98.1$, 21.0, 0.21; HREI m/z calcd. for $\mathrm{C}_{19} \mathrm{H}_{22} \mathrm{O}_{2} \mathrm{Si}: 310.1389(\mathrm{M})^{+}$; found: 310.1383 .

\section{4-[(1E,3Z,5E)-3-Methyl-8-(trimethylsilyl)octa-1,3,5-trien-7-ynyl]phenyl-4-methylbenzenesulfon-}

ate (22). The crude product was loaded on silca gel (doped with triethylamine, $2 \% \mathrm{v} / \mathrm{v}$ ) and eluted with hexanes: EtOAc 5:1. Purified 3-(Z)-trienyne 22, $\mathrm{R}_{\mathrm{f}}=0.32$ (PMA stain), was obtained as an oil; $64 \% ;{ }^{1} \mathrm{H}$ 
$\operatorname{NMR}\left(300 \mathrm{MHz}, \mathrm{CDCl}_{3}\right) \delta 7.63(\mathrm{~d}, \mathrm{~J}=7.9 \mathrm{~Hz}, 2 \mathrm{H}) ; 7.28(\mathrm{~d}, \mathrm{~J}=8.8 \mathrm{~Hz}, 2 \mathrm{H}) ; 7.24(\mathrm{~d}, \mathrm{~J}=7.4 \mathrm{~Hz}, 2 \mathrm{H})$; $7.20(\mathrm{~d}, \mathrm{~J}=16.3 \mathrm{~Hz}, 1 \mathrm{H}) ; 7.11(\mathrm{dd}, \mathrm{J}=11.9,15.4 \mathrm{~Hz}, 1 \mathrm{H}) ; 6.83(\mathrm{~d}, \mathrm{~J}=8.3 \mathrm{~Hz}, 2 \mathrm{H}) ; 6.44(\mathrm{~d}, \mathrm{~J}=15.8$ Hz, 1H); 6.00 (d, J = 11.4 Hz, 1H); 5.57 (d, J = 15.4 Hz, 1H); 2.37 (s, 3H); 1.93 (s, 3H); 0.15 (s, 9H); ${ }^{13} \mathrm{C}$ NMR $\left(75 \mathrm{MHz}, \mathrm{CDCl}_{3}\right) \delta 149.1,145.6,137.6,136.7,136.5,132.5,130.2,130.0,129.1,128.7$, $127.9,110.9,105.2,98.5,21.9,20.8,0.15$.

Trimethyl[(3E,5Z,7E)-6-methyl-8-(2,6,6-trimethylcyclohex-1-enyl)octa-3,5,7-trien-1-ynyl]silane

(23). The crude product was loaded on silca gel (doped with triethylamine, $2 \% \mathrm{v} / \mathrm{v}$ ) and eluted with hexanes. Purified 3-(Z)-trienyne 23, $\mathrm{R}_{\mathrm{f}}=0.28$ (PMA stain), was obtained as an oil; 86\%; ${ }^{1} \mathrm{H}$ NMR (300 $\left.\mathrm{MHz}, \mathrm{CDCl}_{3}\right) \delta 7.12(\mathrm{dd}, \mathrm{J}=11.7,15.3 \mathrm{~Hz}, 1 \mathrm{H}) ; 6.61(\mathrm{~d}, \mathrm{~J}=15.9 \mathrm{~Hz}, 1 \mathrm{H}) ; 6.24(\mathrm{~d}, \mathrm{~J}=15.9 \mathrm{~Hz}, 1 \mathrm{H}) ;$ $5.97(\mathrm{~d}, \mathrm{~J}=11.7 \mathrm{~Hz}, 1 \mathrm{H}) ; 5.56(\mathrm{~d}, \mathrm{~J}=15.3 \mathrm{~Hz}, 1 \mathrm{H}) ; 2.03(\mathrm{t}, \mathrm{J}=6.3 \mathrm{~Hz}, 2 \mathrm{H}) ; 1,97(\mathrm{~s}, 3 \mathrm{H}) ; 1.73$ (bs, 3H); $1.62(\mathrm{~m}, 2 \mathrm{H}) ; 1.48(\mathrm{~m}, 2 \mathrm{H}) ; 1.03(\mathrm{~s}, 6 \mathrm{H}) ; 0.21(\mathrm{~s}, 9 \mathrm{H}) ; \mathrm{HREI} \mathrm{m} / \mathrm{z}$ calcd. for $\mathrm{C}_{21} \mathrm{H}_{32} \mathrm{Si}: 312.2273(\mathrm{M})^{+}$; found: 312.2263 .

General procedure for desilylation of trienynes (Scheme 4). Potassium carbonate $(0.31 \mathrm{~g}, 2.24$ mmol) was taken up in ethanol $(8.0 \mathrm{~mL})$ and combined with the silyltrienyne $(0.73 \mathrm{mmol})$. The heterogeneous mixture was stirred at $\mathrm{rt}$ while the disappearance of starting material was monitored by TLC. After 4-12 h, the mixture was diluted with hexanes $(5.0 \mathrm{~mL})$ and filtered on paper. The filter cake was rinsed with hexanes and the combined organics were extracted using sat. aqueous $\mathrm{NH}_{4} \mathrm{Cl}$, and water, and then dried over anyhydrous $\mathrm{Na}_{2} \mathrm{SO}_{4}$. The dried organics were concentrated under reduced pressure. The free alkynes prepared were unstable, so after purification they were used immediately in subsequent reactions.

1-[(4E,6E,8E)-6-Methylundeca-4,6,8-trien-10-ynyloxy)methyl]benzene (Desilylation: Table 1, entry A). The crude product was loaded on silica gel (doped with triethylamine, $1 \% \mathrm{v} / \mathrm{v}$ ) and eluted with $\mathrm{Et}_{2} \mathrm{O}$-hexanes 1:20. Purified all-trans-terminal alkyne, $\mathrm{R}_{\mathrm{f}}=0.33$ (PMA stain, $\mathrm{Et}_{2} \mathrm{O}$-hexanes 1:20), was obtained as a yellow oil; 90\%; ${ }^{1} \mathrm{H}$ NMR $\left(400 \mathrm{MHz}, \mathrm{CDCl}_{3}\right) \delta$ 7.37-7.34 (m, Ar-4H), 7.32-7.29 (m, 1H), $7.04(\mathrm{dd}, J=15.2,11.6 \mathrm{~Hz}, 1 \mathrm{H}), 6.11(\mathrm{dd}, J=15.6,0.8 \mathrm{~Hz}, 1 \mathrm{H}), 6.02(\mathrm{~d}, J=11.6 \mathrm{~Hz}, 1 \mathrm{H}), 5.82(\mathrm{dt}, J=$ 
15.2, 7.2 Hz, 1H), $5.57(\mathrm{dd}, J=15.6,2.4 \mathrm{~Hz}, 1 \mathrm{H}), 4.52\left(\mathrm{~s}\right.$, benzylic- $\left.\mathrm{CH}_{2}\right), 3.50\left(\mathrm{t}, J=6.4 \mathrm{~Hz}, \mathrm{CH}_{2}\right), 3.15$ (d, $J=2.4 \mathrm{~Hz}$, alkyne-H), 2.26 (q, $\left.J=7.2 \mathrm{~Hz}, \mathrm{CH}_{2}\right), 1.88\left(\mathrm{~d}, J=0.8 \mathrm{~Hz}, \mathrm{CH}_{3}\right), 1.75$ (app. quint., $J=$ ca. $\left.6.8 \mathrm{~Hz}, \mathrm{CH}_{2}\right) ;{ }^{13} \mathrm{C}$ NMR $\left(100 \mathrm{MHz}, \mathrm{CDCl}_{3}\right) \delta 140.1,138.9,138.8,134.87,131.91,128.6,128.2,127.9$, 127.8, 109.0, 84.1, 80.1, 73.1, 69.8, 29.9, 29.7, 13.2; IR (NaCl, neat): 3292, 3092, 2936, 2856, 2091, 1601, 1495, 1454, 1636, 1203, 1102, 1027, 964, 736, $698 \mathrm{~cm}^{-1} ; \mathrm{MS}(\mathrm{m} / \mathrm{z}$, rel \%): 51(11), 65(22), 77 (20), 91(100), 115(18), 131(927), 142(17), 160(95), 175(98), 266(3); HREI m/z calcd. for $\mathrm{C}_{19} \mathrm{H}_{22} \mathrm{O}$ : $266.1671(\mathrm{M})^{+}$, found: 266.1664 .

\section{1,2-Bis(benzyloxy)-4-((1E,3E,5E)-3-methylocta-1,3,5-trien-7-ynyl)benzene (Desilylation: Table 1,} entry B). The crude terminal alkyne, $\mathrm{R}_{\mathrm{f}}=0.28$ (iodine stain, EtOAc:hexanes 1:20), was obtained as a yellow solid; the semi-pure product was triturated using hexanes $(2 \mathrm{x})$ and semi-pure yellow solid was obtained; $\mathrm{mp}=108-110{ }^{\circ} \mathrm{C}$, at melting the product decomposed to a brown residue; $77 \%$; ${ }^{1} \mathrm{H}$ NMR $\left(400 \mathrm{MHz}, \mathrm{CDCl}_{3}\right) \delta$ 7.49-7.34 (m, 10H), 7.12-7.08 (m, 2H), 6.98- $6.89(\mathrm{~m}, 2 \mathrm{H}), 6.67(\mathrm{~d}, J=16 \mathrm{~Hz}$, 1H), $6.57(\mathrm{~d}, J=15.6 \mathrm{~Hz}, 1 \mathrm{H}), 6.22(\mathrm{~d}, J=12 \mathrm{~Hz}, 1 \mathrm{H}), 5.63(\mathrm{~d}, J=15.6 \mathrm{~Hz}, 1 \mathrm{H}), 5.19\left(\mathrm{~s}, \mathrm{CH}_{2}-\mathrm{O}, 4 \mathrm{H}\right)$, 3.15 (s, alkyne-H), $2.00\left(\mathrm{~s}, \mathrm{CH}_{3}\right) ;{ }^{13} \mathrm{C} \mathrm{NMR}\left(100 \mathrm{MHz}, \mathrm{CDCl}_{3}\right) \delta 149.30,149.24,138.7,137.57$, $137.52,137.30,131.2,130.6,128.8,128.7,128.4,128.1,128.0,127.54,127.46,123.5,121.2,114.9$, 112.9, 108.9, 84.0, 80.2, 71.7, 71.4, 20.9; IR (KBr) 3288, 3034, 2936, 2879, 1513, 1387, 1251, 1128, 1006, 960, 804, 746, $698 \mathrm{~cm}^{-1}$; MS (m/z, rel \%): 57(29), 78(24), 113(11), 132(2), 149 (100), 167(31), 279(16); HREI m/z calcd. for $\mathrm{C}_{29} \mathrm{H}_{26} \mathrm{O}_{2}: 406.1933$ (M) ${ }^{+}$, found: 406.1931.

(3E,5E,7E)-6-Methyltetradeca-3,5,7-trien-1-yne (Desilylation: Table 1, entry E). The crude product was loaded on a plug of silica gel (doped with triethylamine, 1\% v/v) and eluted with hexanes. Purified all-trans-terminal alkyne, $\mathrm{R}_{\mathrm{f}}=0.56$ (PMA stain, hexanes), was obtained as a light yellow oil; $93 \%,{ }^{1} \mathrm{H}$ NMR $\left(500 \mathrm{MHz}, \mathrm{CDCl}_{3}\right) \delta 7.04(\mathrm{dd}, J=11.4,15.3 \mathrm{~Hz}, 1 \mathrm{H}), 6.10(\mathrm{dd}, J=0.52,15.6 \mathrm{~Hz}, 1 \mathrm{H}), 6.02(\mathrm{~d}, J$ $=11.7 \mathrm{~Hz}, 1 \mathrm{H}), 5.84$ (app. dt, $J=7.1,15 \mathrm{~Hz}, 1 \mathrm{H}), 5.56(\mathrm{dd}, J=2.3,15.6 \mathrm{~Hz}, 1 \mathrm{H}), 3.09(\mathrm{~d}, J=2.2 \mathrm{~Hz}$ alkyne-H), 2.14 (app. q, $\left.J=6.7 \mathrm{~Hz}, \mathrm{CH}_{2}\right), 1.89$ (d, $\left.J=1.3 \mathrm{~Hz}, \mathrm{CH}_{3}\right), 1.41\left(\mathrm{~m}, \mathrm{CH}_{2}\right), 1.31(\mathrm{~m}, 6 \mathrm{H}), 0.90$ (app. t, $\left.J=7.1 \mathrm{~Hz}, \mathrm{CH}_{3}\right) ;{ }^{13} \mathrm{C} \mathrm{NMR}\left(100 \mathrm{MHz}, \mathrm{CDCl}_{3}\right) \delta 139.94,138.84,134.10,132.78,127.66$, 
$108.55,83.87,79.69,33.11,31.70,29.37,28.90,22.59,14.01,12.93$; IR (NaCl, neat) 3310, 2956, 2925, 2855, 2093, 1458, $962 \mathrm{~cm}^{-1}$; MS (m/z, rel \%): 53(10), 65(9), 77(6), 91(49), 103(4), 117 (100), 131(47), 145(5), 202(25); HREI m/z calcd. for $\mathrm{C}_{15} \mathrm{H}_{22}: 202.1722(\mathrm{M})^{+}$, found: 202.1716 .

(3E,5Z,7E)-6-Methyltetradeca-3,5,7-trien-1-yne (Desilylation of 14). The crude product was loaded on a plug of silica gel (doped with triethylamine, $1 \% \mathrm{v} / \mathrm{v}$ ) and eluted with hexanes. Purified terminal alkyne, $\mathrm{R}_{\mathrm{f}}=0.51$, was obtained as a light yellow oil; 95\%, ${ }^{1} \mathrm{H} \mathrm{NMR}\left(400 \mathrm{MHz}, \mathrm{CDCl}_{3}\right) \delta 7.17(\mathrm{dd}, J=$ 11.6, 15.2 Hz, 1H), $6.61(\mathrm{dd}, J=0.8,15.5 \mathrm{~Hz}, 1 \mathrm{H}), 5.91(\mathrm{~d}, J=11.6 \mathrm{~Hz}, 1 \mathrm{H}), 5.83$ (app. quint., $J=$ ca. $7.2 \mathrm{~Hz}, 1 \mathrm{H}), 5.48$ (dd, $J=2.0,15.2 \mathrm{~Hz}, 1 \mathrm{H}), 3.05$ (d, $2.4 \mathrm{~Hz}$, alkyne-H), 2.17 (q, $J=7.0 \mathrm{~Hz}, 2 \mathrm{H}), 1.92$ (br. s, $\left.\mathrm{CH}_{3}\right), 1.52-1.40(\mathrm{~m}, 2 \mathrm{H}), 1.34-1.26(\mathrm{~m}, 2 \mathrm{H}), 0.92-0.88(\mathrm{~m}, 2 \mathrm{H}), 0.82$ ( app. t, J = 6.9 Hz, $\left.\mathrm{CH}_{3}\right)$; ${ }^{13} \mathrm{C} \mathrm{NMR}\left(100 \mathrm{MHz}, \mathrm{CDCl}_{3}\right) \delta 138.78,137.68,134.09,126.44,126.19,107.86,83.79,79.31,33.4$, $31.71,29.33,28.93,22.59,20.84,14.08 ; \mathrm{IR}$ (NaCl, neat) 3310, 2960, 2925, 2854, 1460, 959, $591 \mathrm{~cm}^{-1}$; MS (m/z, rel \%): 55(15), 73(21), 91(39), 103(14), 131(44), 145(8), 179(54), 202(27). HREI m/z calcd. for $\mathrm{C}_{15} \mathrm{H}_{22}: 202.1722(\mathrm{M})^{+}$, found: 202.1673 .

1-\{[(4E,6Z,8E)-6-Methylundeca-4,6,8-trien-10-ynyloxy]methyl\}benzene (Desilylation of 16). The crude product was loaded on a plug of silica gel (doped with triethylamine, $1 \% \mathrm{v} / \mathrm{v}$ ) and eluted with hexanes: $\mathrm{Et}_{2} \mathrm{O}$ 1:20. Purified terminal alkyne, $\mathrm{R}_{\mathrm{f}}=0.38$ (PMA stain), was obtained as a light yellow oil; 93\%; ${ }^{1} \mathrm{H}$ NMR (400 MHz, $\left.\mathrm{CDCl}_{3}\right) \delta$ 7.37-7.34 (m, 4H), 7.33-7.27 (m, 1H), 7.16 (dd, $J=15.2,11.6 \mathrm{~Hz}$, $1 \mathrm{H}), 6.62(\mathrm{~d}, J=15.2,1 \mathrm{H}), 5.92(\mathrm{~d}, J=11.6,1 \mathrm{H}), 5.83(\mathrm{dt}, J=15.6,7.2 \mathrm{~Hz}, 1 \mathrm{H}), 5.50(\mathrm{dd}, J=15.2,2.0$ $\mathrm{Hz}, 1 \mathrm{H}), 4.52(\mathrm{~s}, 3 \mathrm{H}), 3.50(\mathrm{t}, J=6.6 \mathrm{~Hz}, 2 \mathrm{H}), 3.07(\mathrm{~d}, J=2.4,1 \mathrm{H}), 2.28(\mathrm{q}, J=6.8 \mathrm{~Hz}, 2 \mathrm{H}), 1.90(\mathrm{~s}$, $\mathrm{CH}_{3}$ ), 1.77 (quint, $\left.J=6.8 \mathrm{~Hz}, 2 \mathrm{H}\right) ;{ }^{13} \mathrm{C} \mathrm{NMR}\left(100 \mathrm{MHz}, \mathrm{CDCl}_{3}\right) \delta 138.70,138.50,137.47,133.04$, $128.36,127.63,127.54,126.94,126.45,108.05,83.73,79.43,72.90,69.57,29.97,29.37,20.82$; IR (NaCl, neat): 3090, 3071, 3031, 1959, 1814, 1527, 1475, 1035, 686, $662 \mathrm{~cm}^{-1}$; MS (m/z, rel \%): 51(13), 71(21), 78(37), 91(100), 105(16), 115(13), 131(26), 142(17), 149(6), 160(4), 175(8), 266(3); HREI m/z calcd. for $\mathrm{C}_{19} \mathrm{H}_{22} \mathrm{O}: 266.1671(\mathrm{M})^{+}$, found: 266.1678 . 


\section{6-[(2Z,4E)-Hepta-2,4-dien-6-yn-2-yl]-1,2,3,4-tetrahydro-1,1,4,4-tetramethyl-7-propoxynaphtha-}

lene (Desilylation of 18). The crude product was loaded on a plug of silica gel (doped with triethylamine, $2 \% \mathrm{v} / \mathrm{v}$ ) and eluted with hexanes:EtOAc 100:1. Purified terminal alkyne, $\mathrm{R}_{\mathrm{f}}=0.18$, was obtained as a yellow solid; 67\%; ${ }^{1} \mathrm{H}$ NMR $\left(300 \mathrm{MHz}, \mathrm{CDCl}_{3}\right) \delta 6.93(\mathrm{~s}, 1 \mathrm{H}) ; 6.77(\mathrm{~s}, 1 \mathrm{H}) ; 6.60(\mathrm{dd}, \mathrm{J}=$ 11.0, 15.6 Hz, 1H); $6.17(\mathrm{~d}, \mathrm{~J}=12.0 \mathrm{~Hz}, 1 \mathrm{H}) ; 5.49(\mathrm{dd}, \mathrm{J}=2.1,15.6 \mathrm{~Hz}, 1 \mathrm{H}) ; 3.88(\mathrm{t}, \mathrm{J}=6.6 \mathrm{~Hz}, 2 \mathrm{H})$; $2.93(\mathrm{~d}, \mathrm{~J}=2.1 \mathrm{~Hz}, 1 \mathrm{H}) ; 2.15(\mathrm{~s}, 3 \mathrm{H}) ; 1.77$ (sextet, J = 6.6 Hz, 2H); $1.69(\mathrm{bs}, 4 \mathrm{H}) ; 1.31(\mathrm{~s}, 6 \mathrm{H}) ; 1.26(\mathrm{~s}$ 6H), $1.01(\mathrm{t}, \mathrm{J}=7.4 \mathrm{~Hz}, 3 \mathrm{H}) ;{ }^{13} \mathrm{C} \mathrm{NMR}\left(75 \mathrm{MHz}, \mathrm{CDCl}_{3}\right) \delta 154.0,145.5,142.8,142.4,136.7,128.8$ $127.6,127.1,109.9,107.2,84.1,75.6,70.0,35.4,35.4,34.7,33.8,32.1,32.0,25.1,22.9,11.0$; HREI m/z calcd. for $\mathrm{C}_{24} \mathrm{H}_{32} \mathrm{O}: 336.2453(\mathrm{M})^{+}$, found: 336.2446 .

General procedure for carboalumination of $9 E$ and $9 Z$ trienynes (Table 5). To a solution of $\mathrm{Cp}_{2} \mathrm{ZrCl}_{2}(0.11 \mathrm{~g}, 0.38 \mathrm{mmol})$ in 1,2-dichloroethane $(4.2 \mathrm{~mL})$ was added $\mathrm{Me}_{3} \mathrm{Al}$ or $\mathrm{Et}_{3} \mathrm{Al}(2 \mathrm{M}$ in hexanes, $1.52 \mathrm{mmol}$ ) at $0{ }^{\circ} \mathrm{C}$ and the mixture was stirred for $5 \mathrm{~min}$ at the same temperature. After this period, a solution of trienyne $(0.38 \mathrm{mmol})$ in 1,2 -dichloroethane $(2.1 \mathrm{~mL})$ was added dropwise and the mixture was stirred at $0{ }^{\circ} \mathrm{C}$ for $30 \mathrm{~min}$ and then allowed to warm to rt. After $2 \mathrm{~h}$, no starting material was observed by TLC and to this mixture was added methyl chloroformate $(0.11 \mathrm{~mL}, 1.4 \mathrm{mmol})$ and it was stirred for $1 \mathrm{~h}$ at $\mathrm{rt}$. The reaction was quenched by addition of water $(10 \mathrm{~mL})$ and then extracted with $\mathrm{Et}_{2} \mathrm{O}(4 \times 20 \mathrm{~mL})$. The combined organics were washed with brine $(2 \times 25 \mathrm{~mL})$, dried over anhydrous $\mathrm{MgSO}_{4}$, filtered on paper and concentrated under vacuum.

(2E,4E,6E,8E)-Methyl 12-(benzyloxy)-3,7-dimethyldodeca-2,4,6,8-tetraenoate (25a). The crude product was loaded directly onto silica gel column (doped with trietylamine, 1\% v/v) and flash chromatographed eluting with hexanes: $\mathrm{Et}_{2} \mathrm{O}(7: 1)$. Purified ester 25a $\left(\mathrm{R}_{\mathrm{f}}=0.35\right)$ was obtained as a yellow oil; 89\%; ${ }^{1} \mathrm{H}$ NMR $\left(400 \mathrm{MHz}, \mathrm{CDCl}_{3}\right) \delta$ 7.37-7.34 (m, 4H), 7.31-7.28 (m, 1H), $6.96(\mathrm{dd}, J=$ 15.2, 11.2 Hz, 1H), $6.28(\mathrm{~d}, J=15.2 \mathrm{~Hz}, 1 \mathrm{H}), 6.15(\mathrm{~d}, J=15.6 \mathrm{~Hz}, 1 \mathrm{H}), 6.08(\mathrm{~d}, J=11.2 \mathrm{~Hz}, 1 \mathrm{H}), 5.82$ (app. dt, $J=15.6,6.8 \mathrm{~Hz}, 1 \mathrm{H}), 5.79(\mathrm{~s}, 1 \mathrm{H}), 4.52(\mathrm{~s}, 2 \mathrm{H}), 3.72\left(\mathrm{~s}, \mathrm{CH}_{3}\right) ; 3.50(\mathrm{t}, J=6.4 \mathrm{~Hz}, 2 \mathrm{H}), 2.36$ (d, $\left.J=1.2 \mathrm{~Hz}, \mathrm{CH}_{3}\right), 2.26(\mathrm{q}, J=7.2 \mathrm{~Hz}, 2 \mathrm{H}), 1.93(\mathrm{~d}, J=1.2 \mathrm{~Hz}, 3 \mathrm{H}), 1.75$ (app. quint, $\left.J=6.8 \mathrm{~Hz}, 2 \mathrm{H}\right)$; 
${ }^{13} \mathrm{C}$ NMR $\left(100 \mathrm{MHz}, \mathrm{CDCl}_{3}\right) \delta 167.8,153.2,139.4,138.8,135.3,135.2,131.7,131.3,129.16,128.6$, 127.9, 127.8, 118.4, 73.1, 69.9, 51.2, 30.0, 29.7, 14.1, 13.3; IR ( $\mathrm{NaCl}$, neat) 3028, 2940, 2856, 1709, 1588, 1440, 1241, 1155, 966, $738 \mathrm{~cm}^{-1}$; MS (m/z, rel \%) 71(15), 81(4), 91(100), 105(13), 119(8), 145(16), 171(13), 199(9), 217(8), 231(3), 240(10), 340(6). HREI m/z calcd. for $\mathrm{C}_{22} \mathrm{H}_{28} \mathrm{O}_{3} 340.2038$ $(\mathrm{M})^{+}$, found: 340.2031 .

(2E,4E,6E,8E)-Methyl 3,7-dimethylpentadeca-2,4,6,8-tetraenoate (25b). The crude product was loaded directly onto silica gel column (doped with trietylamine, $1 \% \mathrm{v} / \mathrm{v}$ ) and flash chromatographed eluting with hexanes: $\mathrm{Et}_{2} \mathrm{O}(3 \%)$. Purified ester $\mathbf{2 5 b}\left(\mathrm{R}_{\mathrm{f}}=0.43\right.$, hexanes: $\left.\mathrm{Et}_{2} \mathrm{O}, 5 \%\right)$ was obtained as a yellow oil; 69\%; ${ }^{1} \mathrm{H}$ NMR $\left(400 \mathrm{MHz}, \mathrm{CDCl}_{3}\right) \delta 6.96(\mathrm{dd}, J=15.2,11.2 \mathrm{~Hz}, 1 \mathrm{H}), 6.26(\mathrm{~d}, J=15.2 \mathrm{~Hz}$, 1H), $6.13(\mathrm{~d}, J=15.6 \mathrm{~Hz}, 1 \mathrm{H}), 6.08(\mathrm{~d}, J=11.2 \mathrm{~Hz}, 1 \mathrm{H}), 5.83(\mathrm{app} \mathrm{dt}, J=15.6,7.2 \mathrm{~Hz}, 1 \mathrm{H}), 5.77(\mathrm{~s}$, 1H), 3.7 (s, 3H), 2.34 (d, $J=1.2 \mathrm{~Hz}, 3 \mathrm{H}), 2.14$ (q, $J=7.2 \mathrm{~Hz}, 2 \mathrm{H}), 1.93(\mathrm{~s}, 3 \mathrm{H}), 1.43-1.37$ (m, 2H), 1.34-1.25 (m, 8H), $0.89(\mathrm{t}, J=7.2 \mathrm{~Hz}, 3 \mathrm{H}) ;{ }^{13} \mathrm{C} \mathrm{NMR}\left(100 \mathrm{MHz}, \mathrm{CDCl}_{3}\right) \delta 167.8,153.3,139.6,135.1$, 134.6, 132.8, 131.3, 128.9, 118.21, 51.19, 33.39, 31.94, 29.63, 29.14, 22.83, 14.31, 14.02, 13.32 IR ( $\mathrm{NaCl}$, neat): 2925, 2855, 1714, 1590, 1433, 1353, 1240, 1150, $964 \mathrm{~cm}^{-1}$; MS (m/z, rel \%): 55(38), 69 (18), 78(42), 91(55), 105(53), 112(33), 119(53), 131(82), 145(76), 159(95), 173(20), 199(61), 205(11), 217(100), 224(15), 245(15), 261(9), 276(86); HREI m/z calcd. for $\mathrm{C}_{18} \mathrm{H}_{28} \mathrm{O}_{2}: 276.2029$ (M) ${ }^{+}$, found: 276.2083.

(2E,4E,6E,8E)-Methyl 12-(benzyloxy)-3-ethyl-7-methyldodeca-2,4,6,8-tetraenoate (25c). Following the general procedure for carboalumination (using $\mathrm{Et}_{3} \mathrm{Al}$, the reaction was complete in $45 \mathrm{~min}$ ), the crude product was loaded directly onto a silica gel column (doped with trietylamine, $1 \% \mathrm{v} / \mathrm{v}$ ) and flash chromatographed eluting with hexanes: $\mathrm{Et}_{2} \mathrm{O}$ 7:1. Purified ester $25 \mathbf{c}\left(\mathrm{R}_{\mathrm{f}}=0.63\right)$ was obtained as a yellow oil; 80\%; ${ }^{1} \mathrm{H}$ NMR (400 MHz, $\left.\mathrm{CDCl}_{3}\right) \delta$ 7.38-7.33 (m, 4H), 7.32-7.28 (m, 1H), $6.98(\mathrm{dd}, J=15.2,11.2$ Hz, 1H), 6.18 (app. d, $J=15.2 \mathrm{~Hz}, 1 \mathrm{H}), 6.16$ (app. d, $J=15.6 \mathrm{~Hz}, 1 \mathrm{H}), 6.10$ (d, $J=11.6 \mathrm{~Hz}, 1 \mathrm{H}), 5.83$ (dt, $J=15.6,7.2 \mathrm{~Hz}, 1 \mathrm{H}), 5.73(\mathrm{~s}, 1 \mathrm{H}), 4.52(\mathrm{~s}, 2 \mathrm{H}), 3.72(\mathrm{~s}, 3 \mathrm{H}), 3.51(\mathrm{t}, J=6.4 \mathrm{~Hz}, 2 \mathrm{H}), 2.87$ (q, $J=$ $7.6 \mathrm{~Hz}, 2 \mathrm{H}), 2.27$ (q, $J=7.2 \mathrm{~Hz}, 2 \mathrm{H}), 1.93(\mathrm{~d}, J=0.2 \mathrm{~Hz}, 3 \mathrm{H}$ ), 1.76 (app. quint, $J=6.8 \mathrm{~Hz}, 2 \mathrm{H}) .1 .15$ (t, 
$J=7.6 \mathrm{~Hz}, 3 \mathrm{H}) ;{ }^{13} \mathrm{C} \mathrm{NMR}\left(100 \mathrm{MHz} . \mathrm{CDCl}_{3}\right) \delta 167.3,159.7,139.3,138.8,135.2,133.9,131.6,130.9$, $129.3,128.5,127.9,127.8,117.2,73.1,69.8,51.2,30.0,29.7,21.2,14.4,13.3$; IR ( $\mathrm{NaCl}$, neat): 2937, 2853, 1710, 1584, 1216, 1151, $966 \mathrm{~cm}^{-1}$; MS (m/z, rel \%): 71(15), 81(5), 91(100), 105(12), 119(9), 145(9), 159(11), 185(12), 203(9), 219(2), 231(11), 245(3), 263(12), 354(7). HREI m/z calcd. for $\mathrm{C}_{23} \mathrm{H}_{30} \mathrm{O}_{3}: 354.2195(\mathrm{M})^{+}$, found: 354.2179 .

(2E,4E,6Z,8E)-Methyl 3,7-dimethylpentadeca-2,4,6,8-tetraenoate (25d). The crude was loaded directly onto silica gel column (doped with trietylamine, $1 \% \mathrm{v} / \mathrm{v}$ ) and flash chromatographed eluting with hexanes: $\mathrm{Et}_{2} \mathrm{O}(3 \%)$. Purified ester, $\mathrm{R}_{\mathrm{f}}=0.48$, hexanes: $\mathrm{Et}_{2} \mathrm{O}, 3 \%$ ) was obtained as a yellow liquid; 81\%; ${ }^{1} \mathrm{H}$ NMR $\left(500 \mathrm{MHz}, \mathrm{CDCl}_{3}\right) \delta 7.09(\mathrm{dd}, J=11.5,15.0 \mathrm{~Hz}, 1 \mathrm{H}), 6.66(\mathrm{~d}, J=15.4 \mathrm{~Hz}, 1 \mathrm{H}), 6.20(\mathrm{~d}$, $J=15.2 \mathrm{~Hz}, 1 \mathrm{H}$ ), 5.98 (d, $J=11.6 \mathrm{~Hz}, 1 \mathrm{H}), 5.85$ (app. quint, $J=$ ca. $7.1 \mathrm{~Hz}, 1 \mathrm{H}$ ), 5.76 (br. s, $1 \mathrm{H}$ ), 3.70 (s, $\left.\mathrm{OCH}_{3}\right), 2.35\left(\mathrm{~d}, J=1.2 \mathrm{~Hz}, \mathrm{CH}_{3}\right), 2.19\left(\mathrm{q}, J=7.0 \mathrm{~Hz}, \mathrm{CH}_{2}\right), 1.92\left(\mathrm{~s}, \mathrm{CH}_{3}\right), 1.48-1.39(\mathrm{~m}, 2 \mathrm{H}), 1.36-$ $1.25(\mathrm{~m}, 6 \mathrm{H}), 0.89$ (app. t, $J=$ ca. $\left.7.0 \mathrm{~Hz}, \mathrm{CH}_{3}\right) ;{ }^{13} \mathrm{C} \mathrm{NMR}\left(100 \mathrm{MHz}, \mathrm{CDCl}_{3}\right) \delta 167.8,153.3,138.4$, $134.5,134.4,130.1,127.4,126.8,118.2,51.2,33.7,32.0,29.7,29.2,22.8,21.3,14.3,14.1 ; \mathrm{IR}(\mathrm{NaCl}$, neat) $2927,2855,1714,1595,1434,1355,1242,1157,961 \mathrm{~cm}^{-1}$; MS (m/z, rel \%): 55(37), 77(24), 91(48), 119(49), 131(73), 145(63), 159(81), 191(55), 217(100), 245(13), 276(71); HREI m/z calcd. for $\mathrm{C}_{18} \mathrm{H}_{28} \mathrm{O}_{2} 276.2089(\mathrm{M})^{+}$, found 276.2079 .

(2E,4E,6Z,8E)-Methyl 12-(benzyloxy)-3,7-dimethyldodeca-2,4,6,8-tetraenoate (25e). The crude product was loaded directly onto silica gel column (doped with triethylamine, $1 \% \mathrm{v} / \mathrm{v}$ ) and flash chromatographed eluting with hexanes: $\mathrm{Et}_{2} \mathrm{O}$ 7:1. Purified ester $\left(\mathrm{R}_{\mathrm{f}}=0.32\right)$ was obtained as a yellow oil; 65\%; ${ }^{1} \mathrm{H}$ NMR (400 MHz, $\left.\mathrm{CDCl}_{3}\right) \delta$ 7.36-7.35 (m, 4H), 7.31-7.28 (m, 1H), $7.09(\mathrm{dd}, J=15.2,11.2 \mathrm{~Hz}$, 1H), $6.7(\mathrm{~d}, J=15.2 \mathrm{~Hz}, 1 \mathrm{H}), 6.22(\mathrm{~d}, J=15.2 \mathrm{~Hz}, 1 \mathrm{H}), 6.0(\mathrm{~d}, J=11.2 \mathrm{~Hz}, 1 \mathrm{H}), 5.85(\mathrm{dt}, J=15.2,7.2$ $\mathrm{Hz}, 1 \mathrm{H}), 5.78(\mathrm{~s}, 1 \mathrm{H}), 4.52(\mathrm{~s}, 2 \mathrm{H}), 3.72(\mathrm{~s}, 3 \mathrm{H}), 3.52(\mathrm{t}, J=6.4 \mathrm{~Hz}, 2 \mathrm{H}), 2.36$ (app. d, $J=1.2 \mathrm{~Hz}, 3 \mathrm{H})$, 2.32 (app. q, $J=7.2 \mathrm{~Hz}, 2 \mathrm{H}), 1.93(\mathrm{~s}, 3 \mathrm{H}), 1.82-1.75(\mathrm{~m}, 2 \mathrm{H}) ;{ }^{13} \mathrm{C} \mathrm{NMR}\left(100 \mathrm{MHz}, \mathrm{CDCl}_{3}\right) \delta 167.8$ 153.3, 138.7, 138.11, 134.60, 133.3, 129.3., 128.6, 127.84, 127.79, 127.68, 127.29, 119.3, 73.2, 69.9, 51.2, 30.3, 29.8, 21.3, 14.1; IR (NaCl, neat) 2937, 2854, 1708, 1595, 1438, 1356, 1240, 1154, 1023, 962 , 
737, $697 \mathrm{~cm}^{-1}$; MS (m/z, rel \%): 79(9), 91(100), 105(11), 119(8), 131(11), 145(15), 171(14), 199(9),

217(10), 231(3), 249(9), 340(3); HREI m/z calcd. for $\mathrm{C}_{22} \mathrm{H}_{28} \mathrm{O}_{3}: 340.2038(\mathrm{M})^{+}$, found: 340.2043 .

\section{(2E,4E,6Z)-Methyl 7-(1,2,3,4-tetrahydro-1,1,4,4-tetramethyl-6-propoxynaphthalen-7-yl)-3-}

methyl-octa-2,4,6-trienoate (25f). The crude product was loaded directly onto a silica gel column (doped with trietylamine, $1 \% \mathrm{v} / \mathrm{v}$ ) and flash chromatographed eluting with hexanes:Et $2 \mathrm{O}(5 \%)$. Purified ester $\left(\mathrm{R}_{\mathrm{f}}=0.40\right.$, hexanes:Et $\left.2 \mathrm{O}, 10 \%\right)$ was obtained as a yellow oil; $91 \% ;{ }^{1} \mathrm{H}_{\mathrm{NMR}}\left(400 \mathrm{MHz}, \mathrm{CDCl}_{3}\right) \delta$ $6.97(\mathrm{~s}, 1 \mathrm{H}), 6.8(\mathrm{~s}, 1 \mathrm{H}), 6.63(\mathrm{dd}, J=15.6,11.2 \mathrm{~Hz}, 1 \mathrm{H}), 6.21$ (app.dd, $J=11.2,1.2 \mathrm{~Hz}, 1 \mathrm{H}), 6.22(\mathrm{~d}, J$ $=15.6,1 \mathrm{H}), 5.73(\mathrm{~s}, 1 \mathrm{H}), 3.90(\mathrm{t}, J=6.8 \mathrm{~Hz}, 2 \mathrm{H}), 3.67(\mathrm{~s}, 3 \mathrm{H}), 2.2(\mathrm{~s}, 3 \mathrm{H}), 2,15\left(\mathrm{~d}, J=1.2 \mathrm{~Hz}, \mathrm{CH}_{3}\right)$, 1.77 (app. sextet, $J=7.6 \mathrm{~Hz}, 2 \mathrm{H}), 1.68(\mathrm{~s}, 4 \mathrm{H}), 1.31(\mathrm{~s}, 6 \mathrm{H}), 1.24(\mathrm{~s}, 6 \mathrm{H}), 1.01(\mathrm{t}, J=7.6,3 \mathrm{H}) ;{ }^{13} \mathrm{C}$ NMR $\left(100 \mathrm{MHz}, \mathrm{CDCl}_{3}\right) \delta 167.91,154.21,153.86,145.61,143.59,136.5,133.72,133.61,129.39,128.02$ $127.42,117.61,109.80,69.9,51.13,35.40,35.32,34.72,33.84,32.13,31.99,25.00,22.93,13.93$, 11.02; IR (NaCl, neat): 2960, 1714, 1603, 1240, 1154, 968, $733 \mathrm{~cm}^{-1} ; \mathrm{MS}(\mathrm{m} / \mathrm{z}$, rel \%): 55(7), 69(12), 95(6), 111(13), 121(6), 133(11), 165(6), 197(5), 215(16), 231(100), 246(26), $273(23), 293(7), 309(4)$, 321(20), 336(5), 363(8), 378(6), 395(21), 410(31); HREI m/z calcd. for $\mathrm{C}_{27} \mathrm{H}_{38} \mathrm{O}_{3} 410.2821$ (M) ${ }^{+}$, found: 410.2816

(4E,6E,8E)-Methyl 12-(benzyloxy)-7-methyldodeca-4,6,8-trien-2-ynoate (26). A $100 \mathrm{~mL}$ oven dried round bottom flask fitted with a Teflon-coated stir bar was charged with the all-trans-trienyne 30 (0.69 g, $2.59 \mathrm{mmol})$ and topped with a rubber septum. Addition of THF (10 mL) gave a yellow solution which was cooled to $-78{ }^{\circ} \mathrm{C}$ under argon, and then treated dropwise over 1 min with BuLi $(2.55 \mathrm{M}$ in hexanes, $1.2 \mathrm{~mL}, 3.1 \mathrm{mmol})$. The resulting dark colored mixture was stirred for $5 \mathrm{~min}$, then treated with methyl chloroformate (neat, $0.46 \mathrm{~mL}, 5.64 \mathrm{mmol}$ ); the addition was made rapidly (5-10 seconds) using a plastic syringe. The solution was stirred and warmed to $0{ }^{\circ} \mathrm{C}$ over $30 \mathrm{~min}$, and quenched by addition of sat. aqueous $\mathrm{NH}_{4} \mathrm{Cl}(50 \mathrm{~mL})$, and then extracted using $\mathrm{Et}_{2} \mathrm{O}$ :hexanes, 1:10 (4 x $\left.15 \mathrm{~mL}\right)$. The combined organic layers were washed with brine $(2 \times 10 \mathrm{~mL})$ and dried over anhydrous $\mathrm{Na}_{2} \mathrm{SO}_{4}$. The solution was filtered on paper and concentrated under reduced pressure leaving an amber residue. The 
crude product was loaded on silica gel (doped with triethylamine, $1 \% \mathrm{v} / \mathrm{v}$ ) and eluted with $\mathrm{Et}_{2} \mathrm{O}$ :hexanes 1:20. Purified 26, $\mathrm{R}_{\mathrm{f}}=0.29$ (vanillin stain, $\mathrm{Et}_{2} \mathrm{O}$ :hexanes 1:7), was obtained ( $0.81 \mathrm{~g}$, 96\%) as a yellow oil. ${ }^{1} \mathrm{H}$ NMR $\left(400 \mathrm{MHz}, \mathrm{CDCl}_{3}\right) \delta$ 7.36-7.24 (complex m, $\left.6 \mathrm{H}\right), 6.12(\mathrm{~d}, J=15.5 \mathrm{~Hz}$, 1H), 6.07 (d, $J=11.6 \mathrm{~Hz}, 1 \mathrm{H}), 5.92$ (app. dt., $J=$ ca. $7.4,15.2 \mathrm{~Hz}, 1 \mathrm{H}), 5.62(\mathrm{~d}, J=15.6 \mathrm{~Hz}, 1 \mathrm{H}), 4.51$ (s, benzylic- $\mathrm{CH}_{2}$ ), $3.8\left(\mathrm{~s}, \mathrm{OCH}_{3}\right.$ ), 3.49 (t, $J=6.4 \mathrm{~Hz}, \mathrm{CH}_{2}$ ), 2.27 (app. q, $J=7.2 \mathrm{~Hz}, \mathrm{CH}_{2}$ ), 1.90 (d, $J=$ $1.2 \mathrm{~Hz} \mathrm{CH}_{3}$ ), 1.75 (app. q, $\left.\left.J=6.4 \mathrm{~Hz}, \mathrm{CH}_{2}\right) ;{ }^{13} \mathrm{C} \mathrm{NMR} \mathrm{(125} \mathrm{MHz,} \mathrm{CDCl}_{3}\right) \delta 154.77,144.81,142.18$, $138.70,134.58,133.89,128.54,127.80,127.76,127.73,106.29,87.82,83.06,73.08,69.70,52.81$, 29.97, 29.50, 13.30; IR (NaCl, neat) 3028, 2949, 2860, 2186, 1708, 1595, 1434, 1252, 1099, 965, 746, 698, 459, $437 \mathrm{~cm}^{-1}$; MS (m/z, rel \%): 71 (14), 91 (100), 105 (8), 129 (13), 155 (12), 189 (7), 233 (6), 324 (1); HREI m/z calcd. for $\mathrm{C}_{21} \mathrm{H}_{24} \mathrm{O}_{3}: 324.1725$ (M) ${ }^{+}$, found: 324.1733 .

(2Z,4E,6E,8E)-Methyl 12-(benzyloxy)-3,7-dimethyldodeca-2,4,6,8-tetraenoate (27). A flame dried $10 \mathrm{~mL}$ round bottom flask fitted with a Teflon-coated stir bar was cooled under a stream of argon, charged with CuI $(0.014 \mathrm{~g}, 0,074 \mathrm{mmol})$ and topped with a rubber septum. THF $(0.7 \mathrm{~mL})$ was added and the slurry was cooled to $-30{ }^{\circ} \mathrm{C}$ and treated dropwise with MeLi $(0.102 \mathrm{~mL}, 0.154 \mathrm{mmol})$. The heterogeneous mixture was carefully warmed to $0{ }^{\circ} \mathrm{C}$ for $3 \mathrm{~min}$ then recooled to $-30{ }^{\circ} \mathrm{C}$. The cuprate solution was cooled to $-78{ }^{\circ} \mathrm{C}$ resulting in a heterogeneous solution, which was treated dropwise with a solution of acetylenic ester $26(0.024 \mathrm{~g}, 0.074 \mathrm{mmol})$ in THF $(0.28 \mathrm{~mL})$ using a syringe. The solution was stirred for $20 \mathrm{~min}$ at $-78^{\circ} \mathrm{C}$, then $\mathrm{MeOH}(0.5 \mathrm{~mL})$ was added. The residue was warmed to about 0 ${ }^{\circ} \mathrm{C}$ and and poured into a separatory funnel containing sat. aq. $\mathrm{NH}_{4} \mathrm{Cl}(5 \mathrm{~mL})$, and then extracted with $\mathrm{Et}_{2} \mathrm{O}(4 \times 5 \mathrm{~mL})$. The combined organic layers were washed using water and brine, and then dried over anhydrous $\mathrm{Na}_{2} \mathrm{SO}_{4}$. The dried organics were concentrated under reduced pressure leaving a yellow residue. The crude product was loaded on silica gel (doped with triethylamine, $1 \% \mathrm{v} / \mathrm{v}$ ) and eluted using $\mathrm{Et}_{2} \mathrm{O}$ :hexanes 1:8. Purified 27, $\mathrm{R}_{\mathrm{f}}=0.43$ (vanillin stain), $\mathrm{Et}_{2} \mathrm{O}$ :hexanes 7:1, was obtained ( $0.022 \mathrm{~g}, 88 \%$ ) as a yellow oil; ${ }^{1} \mathrm{H}$ NMR $\left(500 \mathrm{MHz}, \mathrm{CDCl}_{3}\right) \delta 7.77(\mathrm{~d}, J=15.2 \mathrm{~Hz}, 1 \mathrm{H}), 7.39-7.33$ (m, Ar-4H), 7.32$7.28(\mathrm{~m}, \mathrm{Ar}-\mathrm{H}), 6.95$ (dd, $J=11.6,15.2 \mathrm{~Hz}, 1 \mathrm{H}), 6.20$ (app. d, $J=$ ca. $11 \mathrm{~Hz}, 1 \mathrm{H}), 6.15$ (app. d, $J=14,8$ 
$\mathrm{Hz}, 1 \mathrm{H}), 5,82(\mathrm{dt}, J=15.6,7.2 \mathrm{~Hz}, 1 \mathrm{H}), 5.65(\mathrm{~s}, 1 \mathrm{H}), 4.52(\mathrm{~s}, 2 \mathrm{H}), 3.72(\mathrm{~s}, 3 \mathrm{H}) ; 3.50(\mathrm{t}, J=6,4 \mathrm{~Hz}, 2 \mathrm{H})$, $2,26(\mathrm{q}, J=7,2 \mathrm{~Hz}, 2 \mathrm{H}), 2,07(\mathrm{~d}, J=0,8 \mathrm{~Hz}, 3 \mathrm{H}), 1,93(\mathrm{~s}, 3 \mathrm{H}), 1.76$ (app. quint, $J=6.4 \mathrm{~Hz}, 2 \mathrm{H}) .{ }^{13} \mathrm{C}$ NMR $\left(100 \mathrm{MHz}, \mathrm{CDCl}_{3}\right) \delta 167.8,153.3,138.7,138.11,134.6,133.3,130.0,128.6,127.8,127.78$, 127.68, 127.3, 118.3, 73.2, 69.9, 51.2, 30.4, 29.7, 21.28, 14.1; IR (NaCl, neat) 3403, 3301, 3029, 2946, 2198, 2074, 1710, 1590, 1453, 1364, 1240, 1158, 1103, 974, 737, $698 \mathrm{~cm}^{-1}$; MS (m/z, rel \%): 51 (6), 65(14), 77(17), 91(100), 105(13), 131(10), 145(13), 159(11), 199(6), 249(5), 340(2); HREI m/z calcd. for $\mathrm{C}_{22} \mathrm{H}_{28} \mathrm{O}_{3}: 340.2038(\mathrm{M})^{+}$, found: 340.2045 .

(2Z,4E,6E,8E)-Methyl 12-(benzyloxy)-3-butyl-7-methyldodeca-2,4,6,8-tetraenoate (28). A flame dried $25 \mathrm{~mL}$ round bottom flask fitted with a Teflon-coated stir bar was cooled under a stream of argon, charged with $\mathrm{CuI}(79 \mathrm{mg}, 0.42 \mathrm{mmol})$, and topped with a rubber septum. THF (2.0 mL) was added and the mixture was cooled to $-78{ }^{\circ} \mathrm{C}$ and treated dropwise over one minute with $n$-BuLi $(2.49 \mathrm{M}$, hexanes $)$ $(0.33 \mathrm{~mL}, 0.82 \mathrm{mmol})$. The alkyne $(100 \mathrm{mg}, 0.31 \mathrm{mmol})$ in $2.0 \mathrm{~mL}$ THF was added dropwise. The reaction was stirred for $3 \mathrm{~h}$ at $-78{ }^{\circ} \mathrm{C}$ then quenched by fast addition of methanol $(2.0 \mathrm{~mL})$ and warmed to $0{ }^{\circ} \mathrm{C}$. The mixture was diluted with $\mathrm{Et}_{2} \mathrm{O}(20 \mathrm{~mL})$ and poured into a separatory funnel containing sat. $\mathrm{NH}_{4} \mathrm{Cl}(\mathrm{aq})(15 \mathrm{~mL})$ and extracted using $\mathrm{Et}_{2} \mathrm{O}(3 \times 20 \mathrm{~mL})$. The combined organic layers were washed using water, and brine, and then dried over anhydrous $\mathrm{Na}_{2} \mathrm{SO}_{4}$. The dried organics were concentrated under reduced pressure leaving a yellow residue. The crude product was loaded on silica gel (doped with triethylamine, 3\% v/v) and eluted using $\mathrm{Et}_{2} \mathrm{O}$-hexanes 1:7. Purified 28, $\mathrm{R}_{\mathrm{f}}=0.41$ (vanillin stain), $\mathrm{Et}_{2} \mathrm{O}$-hexanes $1: 7$, was obtained $(0.107 \mathrm{~g}, 0.28 \mathrm{mmol}, 90 \%)$ as a yellow oil. ${ }^{1} \mathrm{H} \mathrm{NMR}(500 \mathrm{MHz}$, $\left.\mathrm{CDCl}_{3}\right) \delta 7.71(\mathrm{~d}, J=15.4 \mathrm{~Hz}, 1 \mathrm{H}), 7.36($ app. d, $J=4.5 \mathrm{~Hz}, 4 \mathrm{H}), 7.31(\mathrm{~m}, 1 \mathrm{H}), 6.98(\mathrm{dd}, J=15.3,11.6$ Hz, 1H), 6.19 (m, 2H), 5.81 (app. quint., $J=7.5 \mathrm{~Hz}, 1 \mathrm{H}), 5.65$ (br. s, 1H), 4.52 (s, 2H), 3.72 (s, 3H), $3.50(\mathrm{t}, J=5.9 \mathrm{~Hz}, 2 \mathrm{H}), 2.41(\mathrm{t}, J=6.5 \mathrm{~Hz}, 2 \mathrm{H}), 2.26(\mathrm{q}, J=6.9 \mathrm{~Hz}, 2 \mathrm{H}), 1.92(\mathrm{~s}, 3 \mathrm{H}), 1.76(\mathrm{p}, J=7.0$ Hz, 2H), 1.54 (app. quint., $J=7.0 \mathrm{~Hz}, 2 \mathrm{H}), 1.39$ (sext., $J=6.9 \mathrm{~Hz}, 2 \mathrm{H}), 0.95$ (t, $J=7.5 \mathrm{~Hz}, 3 \mathrm{H})$; ${ }^{13} \mathrm{C}$ NMR $\left(125 \mathrm{MHz}, \mathrm{CDCl}_{3}\right) \delta 167.1,156.0,139.2,138.7,135.3,131.8,131.3,130.1,128.6,128.5,127.8$, 127.6, 115.4, 73.0, 69.7, 51.0, 34.0, 31.8, 29.9, 29.6, 22.7, 14.0, 13.2; IR (NaCl, neat) 3059, 3028, 
$2947,2867,1711,1590,1564,1459,1434,1378,1365,1235,1197,1156,1104,1029,973,736,699$

$\mathrm{cm}^{-1}$; HREI $\mathrm{m} / \mathrm{z}$ calcd. for $\mathrm{C}_{25} \mathrm{H}_{34} \mathrm{O}_{3} 382.2508(\mathrm{M})^{+}$, found 382.2498 .

(2Z,4E,6E,8E)-Methyl 12-(benzyloxy)-7-methyl-3-(trimethylstannyl) dodeca-2,4,6,8-tetraenoate

(29). A $25 \mathrm{~mL}$ round bottom flask fitted with a Teflon-coated stir bar was flame dried and cooled under a stream of argon and topped with a rubber septum, then charged with hexamethylditin $(1.0 \mathrm{~g}, 3.05$ mmol) using THF $(2.0 \mathrm{~mL})$ to aid the transfer. The stirred mixture was cooled to $-20{ }^{\circ} \mathrm{C}$ and methyllithium $\left(1.5 \mathrm{M}, \mathrm{Et}_{2} \mathrm{O}\right)(2.0 \mathrm{~mL}, 3.1 \mathrm{mmol})$ was added dropwise over 1 min followed by stirring at $-20{ }^{\circ} \mathrm{C}$ for $30 \mathrm{~min}$. The mixture was cooled to $-78{ }^{\circ} \mathrm{C}$ and copper(I) bromide-dimethyl sulfide complex (0.32 g, $1.53 \mathrm{mmol})$ was added as a solid while flushing the flask with argon, followed by stirring for $35 \mathrm{~min}$ and formation of a brown heterogeneous mixture. The mixture was cooled to -100 ${ }^{\circ} \mathrm{C}$ and treated with a precooled solution of acetylenic ester $26(0.48 \mathrm{~g}, 1.48 \mathrm{mmol})$ plus dry methanol $(85 \mu \mathrm{L})$ in THF $(2.1 \mathrm{~mL})$ dropwise using a precooled cannula. The stirred mixture was held at $-100{ }^{\circ} \mathrm{C}$ for $2 \mathrm{~h}$, then warmed to $0{ }^{\circ} \mathrm{C}$ over $35 \mathrm{~min}$, and quenched by addition of water $(10 \mathrm{~mL})$, and then poured into a separatory funnel containing sat. $\mathrm{NH}_{4} \mathrm{Cl}(\mathrm{aq})(15 \mathrm{~mL})$ plus concentrated $\mathrm{NH}_{4} \mathrm{OH}(\mathrm{aq})(3.0 \mathrm{~mL})$. The mixture was left for $30 \mathrm{~min}$ then extracted using EtOAc-hexanes 1:50 ( 3 x $30 \mathrm{~mL})$, and the combined organic layers were washed using brine, (attempts to use water for washing gave an emulsion) and then dried over anhydrous $\mathrm{Na}_{2} \mathrm{SO}_{4}$. The dried organics were filtered on paper and the filtrate was concentrated under reduced pressure leaving a yellow residue. The crude product was loaded on silica gel (doped with triethylamine, $3 \% \mathrm{v} / \mathrm{v}$ ) and eluted using $\mathrm{Et}_{2} \mathrm{O}$-hexanes 1:7, giving recovered acetylenic ester 29 (53 $\mathrm{mg}, 0.16 \mathrm{mmol})$ plus 2-(Z)-methyl product isomer. Semi-purified 2(Z)-29 (32 mg, 43\%) $\mathrm{R}_{\mathrm{f}}=0.35$ (vanillin stain), $\mathrm{Et}_{2} \mathrm{O}$-hexanes 1:7, as a yellow oil. ${ }^{1} \mathrm{H}$ NMR (400 MHz, $\left.\mathrm{CDCl}_{3}\right) \delta 7.93\left(\mathrm{~d}, J=15.2 \mathrm{~Hz},{ }^{3} J_{\mathrm{SnH}}=43.9 \mathrm{~Hz}, 1 \mathrm{H}\right), 7.36-7.32(\mathrm{~m}, 4 \mathrm{H}), 7.31-7.27(\mathrm{~m}, 1 \mathrm{H}), 6.75(\mathrm{dd}, J$ $\left.=15.0,11.2 \mathrm{~Hz},{ }^{3} J_{\mathrm{SnH}}=3.9 \mathrm{~Hz}, 1 \mathrm{H}\right), 6.19$ (br. d, $\left.J=10.7 \mathrm{~Hz}, 1 \mathrm{H}\right), 6.15$ (d, $\left.J=15.6 \mathrm{~Hz}, 1 \mathrm{H}\right), 5.85$ (br. $\mathrm{s}, 1 \mathrm{H}), 5.86-5.77(\mathrm{~m}, 1 \mathrm{H}), 4.51(\mathrm{~s}, 2 \mathrm{H}), 3.72(\mathrm{~s}, 3 \mathrm{H}), 3.45(\mathrm{t}, J=5.1 \mathrm{~Hz}, 2 \mathrm{H}), 2.26$ (quart., $J=5.4 \mathrm{~Hz}$, $2 \mathrm{H}), 1.88(\mathrm{~d}, J=0.9 \mathrm{~Hz}, 3 \mathrm{H}), 1.75$ (quint., $J=5.1 \mathrm{~Hz}, 2 \mathrm{H}), 0.30\left(\mathrm{~s},{ }^{2} J_{\mathrm{SnH}}=26.7 \mathrm{~Hz}, 9 \mathrm{H}\right)$; assignments 
of the C3-trimethylstannanyl group in addition to the 2-(Z)-olefin geometry and the vinyl- $\mathrm{H}-5$ and vinyl- $\mathrm{H}-2$ resonances was confirmed by an nOe enhancement of the $\delta 6.75$ double doublet and $\delta 5.85$ broad singlet when the $\delta 0.30$ singlet was irradiated; assignment of the vinyl-H-4, and the vinyl-H-6 resonance that partially overlapped the vinyl-H-8 resonance, was confirmed by an nOe enhancement of the $\delta 6.19$ broad doublet when the $\delta 7.93$ doublet was irradiated; several impurities were seen and were not included in this assignment, which is tentative and not supported by either ${ }^{13} \mathrm{C}$ NMR or IR spectra; HREI $\mathrm{m} / \mathrm{z}$ calcd. for $\mathrm{C}_{24} \mathrm{H}_{34} \mathrm{O}_{3}{ }^{116} \mathrm{Sn} 486.1525(\mathrm{M})^{+}$, found 486.1516 .

\section{1-(((4E,6E,8E,10E)-11-Iodo-6,10-dimethylundeca-4,6,8,10-tetraenyloxy)methyl)benzene}

$(31 ;$

Scheme 6). To a stirred solution of $\mathrm{Cp}_{2} \mathrm{ZrCl}_{2}(0.021 \mathrm{~g}, 0.0718 \mathrm{mmol})$ in 1,2-dichloroethane (DCE, 1 $\mathrm{mL})$ was added $\mathrm{Me}_{3} \mathrm{Al}(2.0 \mathrm{M}$ in hexanes, $150 \mu \mathrm{L}, 0.300 \mathrm{mmol})$ at $0{ }^{\circ} \mathrm{C}$. After $10 \mathrm{~min}$, to the mixture was added trienyne $30(0.019 \mathrm{~g}, 0.0713 \mathrm{mmol})$ in DCE $(0.5 \mathrm{~mL})$. After $1.75 \mathrm{~h}$, the mixture was treated with $\mathrm{I}_{2}(0.038 \mathrm{~g}, 0.150 \mathrm{mmol})$ in THF $(0.8 \mathrm{~mL})$ and stirring continued with gradual warming to $25^{\circ} \mathrm{C}$ for $17 \mathrm{~h}$. Water $(0.3 \mathrm{~mL})$ was added slowly and the mixture was extracted with $\mathrm{Et}_{2} \mathrm{O}$. The combined organic extracts were washed with water, brine, and dried over anhydrous $\mathrm{Na}_{2} \mathrm{SO}_{4}$, then concentrated in vacuo. Flash chromatography $\left(5 \% \mathrm{Et}_{2} \mathrm{O} /\right.$ hexanes doped with $\left.0.5 \% \mathrm{v} / \mathrm{v} \mathrm{Et} \mathrm{t}_{3} \mathrm{~N}\right)$ afforded iodide $31(0.019$ g, $65 \%$ ) as a yellow oil: ${ }^{1} \mathrm{H}$ NMR (400 MHz, $\left.\mathrm{C}_{6} \mathrm{D}_{6}\right) \delta 7.32$ (app. d, $\left.J=7.6 \mathrm{~Hz}, 1 \mathrm{H}\right), 7.22-7.09(\mathrm{~m}, 4 \mathrm{H})$, $6.54(\mathrm{dd}, J=15.2,11.2 \mathrm{~Hz}, 1 \mathrm{H}), 6.19(\mathrm{~d}, J=15.4 \mathrm{~Hz}, 1 \mathrm{H}), 6.09$ (app. s, 1H), 6.08 (d, $J=15.2 \mathrm{~Hz}, 1 \mathrm{H})$, $5.97(\mathrm{~d}, J=11.6 \mathrm{~Hz}, 1 \mathrm{H}), 5.68(\mathrm{dt}, J=14.8,7,2 \mathrm{~Hz}, 1 \mathrm{H}), 4.34(\mathrm{~s}, 2 \mathrm{H}), 3.32(\mathrm{t}, J=6.2 \mathrm{~Hz}, 2 \mathrm{H}), 2.22(\mathrm{q}$, $J=7.2 \mathrm{~Hz}, 2 \mathrm{H}), 1.84(\mathrm{~s}, 3 \mathrm{H}), 1.71(\mathrm{~s}, 3 \mathrm{H}), 1.70-1.64(\mathrm{~m}, 2 \mathrm{H}) ;{ }^{13} \mathrm{C} \mathrm{NMR}\left(125 \mathrm{MHz},\left(\mathrm{CD}_{3}\right)_{2} \mathrm{CO}\right) \delta$ 147.1, $143.8,140.5,139.3,138.3,136.4,134.1,131.4,130.5,129.6,128.6,127.5,84.3,80.2,73.7,70.6,21.4$ 13.6; IR ( $\mathrm{NaCl}$, neat): 3090, 3071, 3035, 2925, 2855, 1959, 1814, 1478, 1103, 1036, 965, $736 \mathrm{~cm}^{-1}$; MS (m/z, rel \%): 71(11), 77(10), 91(100), 103(10), 105(13), 131(15), 145(18), 408(8); HREI m/z calcd. for $\mathrm{C}_{20} \mathrm{H}_{25} \mathrm{IO}: 408.0943(\mathrm{M})^{+}$, found: 408.0950.

(2Z,4E,6E,8E)-Methyl 12-(benzyloxy)-7-methyldodeca-2,4,6,8-tetraenoate (32). To a stirred solution of trienyne ester $26(0.031 \mathrm{~g}, 0.096 \mathrm{mmol})$ in EtOAc $(5 \mathrm{~mL})$ was added Lindlar's catalyst $(7 \mathrm{mg}, 20 \mathrm{wt}$. 
$\%$ loading) at $25{ }^{\circ} \mathrm{C}$. After $10 \mathrm{~min}$, the mixture was treated with quinoline $(12 \mu \mathrm{L}, 0.102 \mathrm{mmol})$ and stirring was continued for 55 min under a balloon of $\mathrm{H}_{2}(\mathrm{~g})$. The mixture was filtered through Celite, eluting with $\mathrm{Et}_{2} \mathrm{O}$, and concentrated in vacuo. Flash chromatography (doped with $1 \% \mathrm{v} / \mathrm{v} \mathrm{Et}_{3} \mathrm{~N}$ ) eluted with hexanes: $\mathrm{Et}_{2} \mathrm{O}(9: 1)$ afforded the reduced tetraene ester $32(0.024 \mathrm{~g}, 77 \%)$ as a yellow oil; $\mathrm{R}_{\mathrm{f}}=0.30$, (hexanes:Et ${ }_{2} \mathrm{O} 5: 1$ ); ${ }^{1} \mathrm{H}$ NMR (400 MHz, $\mathrm{CDCl}_{3}$ ) $\delta 7.53$ (app. dd, $J=14.8,11.6 \mathrm{~Hz}, 1 \mathrm{H}$ ), 7.37-7.28 (m, 5H), 6.84 (dd, $J=14.8,11.6 \mathrm{~Hz}, 1 \mathrm{H}), 6.68$ (app. t, $J=$ ca. $11.4 \mathrm{~Hz}, 1 \mathrm{H}$ ), 6.17 (app. d, $J=11.2 \mathrm{~Hz}, 1 \mathrm{H}$ ), 6.16 (app. d, $J=15.6 \mathrm{~Hz}, 1 \mathrm{H}), 5.84(\mathrm{dt}, J=15.6,7.2 \mathrm{~Hz}, 1 \mathrm{H}), 5,63(\mathrm{~d}, J=11.2 \mathrm{~Hz}, 1 \mathrm{H}), 4.52(\mathrm{~s}, 2 \mathrm{H})$, 3.75 (s, 3H), 3.50 (t, $J=6.4 \mathrm{~Hz}, 2 \mathrm{H}), 2.27$ (app. q, $J=7.2 \mathrm{~Hz}, 2 \mathrm{H}), 1.92$ (s, $3 \mathrm{H}), 1.76-1.72(\mathrm{~m}, 2 \mathrm{H}) ;{ }^{13} \mathrm{C}$ $\operatorname{NMR}\left(100 \mathrm{MHz}, \mathrm{CDCl}_{3}\right): \delta 167.3,145.9,145.0,140.4,138.7,137.7,135.2,132.1,131.1,129.2,128.0$ 127.2, 116.1, 115.4, 73.1, 69.8, 51.3, 29.9, 13.2; IR (NaCl, neat): 3089, 3071, 3040, 3030, 1960, 1815, 1482, 1173; MS (m/z, rel \%): 71 (15), 91 (100), 105 (11), 131 (11), 185 (6), 256 (7), 295 (9), 326 (25); HREI m/z calcd. for $\mathrm{C}_{21} \mathrm{H}_{26} \mathrm{O}_{3} 326.1882(\mathrm{M})^{+}$, found. 326.1884 . 